\title{
əTopographic Control of Southern Ocean Gyres and the Antarctic Circumpolar Current: A Barotropic Perspective
}

\author{
Ryan D. Patmore, Paul R. Holland, And David R. Munday \\ British Antarctic Survey, Cambridge, United Kingdom \\ Alberto C. NAveira Garabato \\ University of Southampton, Southampton, United Kingdom \\ DAVID P. STEVENS \\ University of East Anglia, Norwich, United Kingdom \\ MiCHAEL P. MEREDITH \\ British Antarctic Survey, Cambridge, United Kingdom
}

(Manuscript received 5 April 2019, in final form 27 September 2019)

\begin{abstract}
In the Southern Ocean the Antarctic Circumpolar Current is significantly steered by large topographic features, and subpolar gyres form in their lee. The geometry of topographic features in the Southern Ocean is highly variable, but the influence of this variation on the large-scale flow is poorly understood. Using idealized barotropic simulations of a zonal channel with a meridional ridge, it is found that the ridge geometry is important for determining the net zonal volume transport. A relationship is observed between ridge width and volume transport that is determined by the form stress generated by the ridge. Gyre formation is also highly reliant on the ridge geometry. A steep ridge allows gyres to form within regions of unblocked geostrophic $(f / H)$ contours, with an increase in gyre strength as the ridge width is reduced. These relationships among ridge width, gyre strength, and net zonal volume transport emerge to simultaneously satisfy the conservation of momentum and vorticity.
\end{abstract}

\section{Introduction}

The Antarctic Circumpolar Current (ACC) and Southern Ocean gyres are key components of the global climate system. The Southern Ocean forms the primary pathway for communication between the Atlantic, Indian, and Pacific Oceans (Talley 2013; Naveira Garabato et al. 2014). Water, heat, salt, and other important tracers are transported zonally between these ocean basins around Antarctica via the ACC (Talley 2013), which has an estimated total volume transport of $173.3 \pm 10.7 \mathrm{~Sv}\left(1 \mathrm{~Sv} \equiv 10^{6} \mathrm{~m}^{3} \mathrm{~s}^{-1}\right)$ (Donohue et al. 2016). A double overturning cell is

¿ Denotes content that is immediately available upon publication as open access.

Corresponding author: Ryan D. Patmore, ryapat30@bas.ac.uk responsible for the meridional exchange of water masses between the Southern Ocean and the basins to the north (Speer et al. 2000). The lower cell of this overturning is reliant on diabatic processes that predominately occur in the vicinity of Southern Ocean gyres (Naveira Garabato et al. 2014) and, thus, gyre dynamics are implicated in overturning activity.

The Southern Ocean has numerous topographic features with scales similar to terrestrial mountain ranges, spanning distances of $O(1000) \mathrm{km}$. Large-scale topographic features are of particular importance in the Southern Ocean (Hughes and Killworth 1995) and are known to have a significant effect on steering the path of the ACC (Gordon et al. 1978; Killworth 1992).

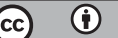

This article is licensed under a Creative Commons Attribution 4.0 license (http://creativecommons.org/ licenses/by/4.0/) 


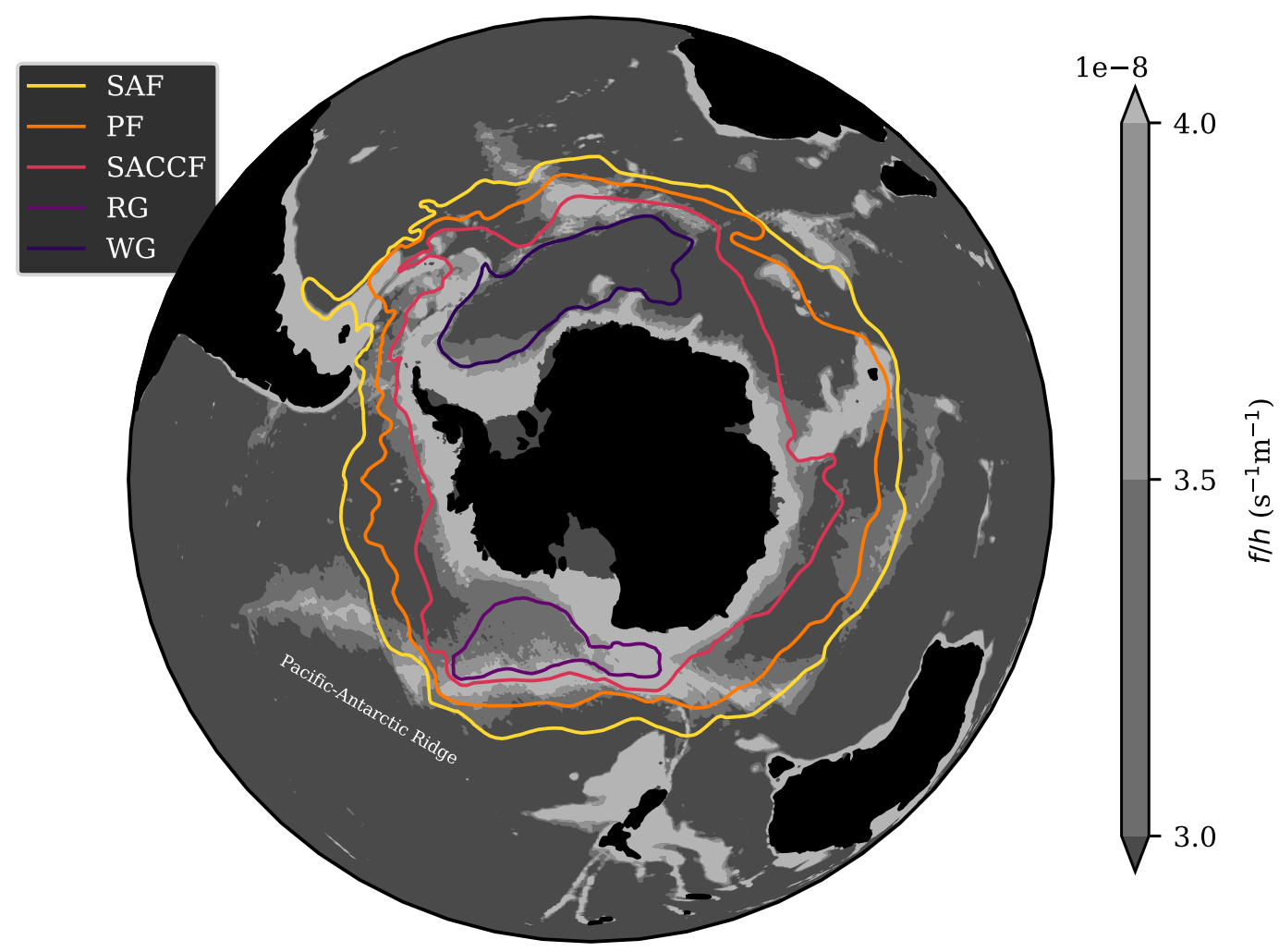

FIG. 1. The $f / h$ for the Southern Hemisphere with ocean fronts and gyre contours overlaid; $f / h$ shading (the numbers are $\times 10^{-8}$ ) is bounded by the $f / h$ values used in Marshall (1995). The three ACC fronts are plotted using data from Orsi et al. (1995) and are defined as: the Subantarctic Front (SAF), the Polar Front (PF), and the Southern Antarctic Circumpolar Current Front (SACCF). The two gyres, the Weddell Gyre (WG) and the Ross Gyre (RG), are plotted using recent satellite data from Armitage et al. (2018).

Figure 1 highlights the effect of topography on the ACC, illustrating the influence of topographic steering. A notable feature that steers the ACC is the PacificAntarctic Ridge, which deflects the current northward over the western flank of the ridge until it passes through the fracture zones to the north.

The primary influence of topographic steering is best described in terms of vorticity and vertical stretching. The context for describing large-scale flow in this way was first outlined by Rossby (1936) and later named in Rossby et al. (1940) as potential vorticity. Flow must conserve potential vorticity, and to leading order it does so by maintaining $f / H$, where $f$ is the Coriolis parameter and $H=h+\eta$ is the water column thickness, with $h$ being the distance from the resting ocean surface to the bottom topography and $\eta$ being the sea surface height ( $\mathrm{SSH}$ ) anomaly. As flow is directed toward the peak of a ridge, it travels equatorward to satisfy conservation of potential vorticity. Flow then reverses direction and travels poleward as the water column thickness increases on the lee side of the ridge. Conservation of potential vorticity means that flow tends to align with contours of $f / H$ (geostrophic contours).

The process of topographic steering has a central role in the momentum balance of the Southern Ocean. Wind stress provides a source of momentum at the surface of the ocean. To satisfy the momentum balance there must be an equal and opposite sink. The meridional deflection of the ACC by topography is associated with form stress, the primary zonal momentum sink balancing the wind stress in the Southern Ocean (Munk and Palmén 1951; Wolff et al. 1991; Stevens and Ivchenko 1997; Masich et al. 2015). Form stress develops where the ACC is subject to inertial effects or bottom stress as it traverses topography (Naveira Garabato et al. 2013). Under these conditions, the ACC deviates from geostrophic contours leading to a lateral offset in $\mathrm{SSH}$ with respect to the topography. A net pressure force across topography associated with this offset in SSH leads to a generation of form stress (Stewart and Hogg 2017). Topography is important in regulating the net zonal transport of the ACC. One aspect of this topographic control is related to form 
stress (Munk and Palmén 1951). A zonal momentum balance between bottom stress and wind stress results in an unrealistically large volume transport in comparison with a balance between form stress and wind stress (Munk and Palmén 1951).

The volume transport through a channel is also sensitive to the proportion of blocked geostrophic contours. In an area of unblocked geostrophic contours, the dynamics are described by a "linear free mode" (Read et al. 1986; Hughes et al. 1999; Tansley and Marshall 2001) and consequently a through channel flow can develop. The space of blocked geostrophic contours emerges through the presence of "landmasses," creating an area where Sverdrup balance dominates and classical gyres form as a result (LaCasce and Isachsen 2010). As ridge height increases, the topography acts to block geostrophic contours and zonal volume transport reduces in strength (Krupitsky and Cane 1994; Wang and Huang 1995; Nadeau and Ferrari 2015). This binary view of the Southern Ocean is only directly applicable to a linear barotropic setting and we emphasize that many other factors add complexity to this simple theory. Much of the ACC, governed by an "almost free mode," drifts into regions of globally blocked geostrophic contours (Hughes et al. 1999). Nevertheless, the simple view that net zonal volume transport is dependent on the level of blocked $f / H$ is a useful construct for this barotropic study.

Linear studies show that the net zonal volume transport in a channel is also dependent on ridge width (Johnson and Hill 1975; Krupitsky and Cane 1994; Wang and Huang 1995). Varying topography in this way does not affect the level of blocked $f / H$ and it isolates the dependence on the ridge geometry itself. These existing studies investigate the linear dynamics in two settings, one where all geostrophic contours are blocked (Krupitsky and Cane 1994; Wang and Huang 1995) and one where there is only partial blocking of geostrophic contours (Johnson and Hill 1975). Localized blocking of geostrophic contours is dynamically distinct from the partially unblocked case because of the way that form stress develops in a linear system. Form stress can only develop where bottom stress curl permits flow to cross geostrophic contours and this process is more readily attained where geostrophic contours are locally blocked (Wang and Huang 1995). However, in the Southern Ocean, few continental boundaries exist for the blocking of geostrophic contours. As a result, it is indicated that a large proportion of the form stress momentum sink occurs over topography that has no association with blocked geostrophic contours (Masich et al. 2015). The lack of requirement for localized blocked geostrophic contours in the Southern Ocean stems from nonlinearity. The form stress is largely governed by topographic Rossby waves (Thompson and Naveira Garabato 2014), which require nonlinear dynamics (McCartney 1976; Johnson 1977). This highlights the importance of understanding the case with locally unblocked geostrophic contours in a nonlinear system.

Progress has been made on the nonlinear case with dynamical discussions given by Treguier and McWilliams (1990), Wolff et al. (1991), and Stevens and Ivchenko (1997). However, unlike the linear case, questions remain around the effects of geometric variations. The relationship between ridge height and volume transport has been investigated in a nonlinear setting by Nadeau and Ferrari (2015) but changes in ridge height affect the level of blocked $f / H$. A nonlinear dynamical understanding of the effects of ridge geometry in a region of unblocked geostrophic contours is currently missing from the literature.

Not only is ridge topography important in determining the ACC volume transport but it is also crucial to Southern Ocean gyre formation. With the prevailing theory of potential vorticity, poleward ACC flow should dominate the eastern flanks of ridges in the Southern Ocean, but this is not uniformly the case. Figure 1 shows that particular locations east of ridge topography are dominated by gyre circulations. In classical gyre theory, western boundary currents are supported by the intersection of geostrophic contours with continental boundaries, which lead to the formation of gyres (see Patmore 2018). Departing from this theory, circulations such as the Ross Gyre occur in the absence of continental boundaries. Instead, these gyres form in the lee of large meridional, submarine topographic features where geostrophic contours are locally unblocked. Modeling results have been presented where gyres form without the need for a western boundary (Krupitsky and Cane 1994; Wang and Huang 1995; Munday et al. 2015). However, the northern boundary in these simulations emulates a continent by blocking geostrophic contours. As a result, the gyres coincide with blocked geostrophic contours and it is not clear this regime is representative of Southern Ocean dynamics, since no northern boundary exists. Nadeau and Ferrari (2015) propose that Southern Ocean gyres can be supported via a threshold of topographic gradients alone. However, their simulations also contain geostrophic contours that are blocked by a northern wall and so the effects of topographic gradients are not isolated.

This study investigates the role of topographic geometry in ACC dynamics and the formation of Southern Ocean gyres in a nonlinear, barotropic setting with unblocked geostrophic contours. Specifically we aim to 
examine the hypothesis of Nadeau and Ferrari (2015) that topographic gradients alone can produce gyres, and we delve deeper into the details of why this might occur. We also explore the unknown effects of topographic geometry on nonlinear barotropic dynamics in unblocked geostrophic contours. Although the system we present permits barotropic eddies and nonlinear effects, it is not in the barotropic eddy-saturated regime demonstrated by Constantinou (2018). We therefore frame the discussion of the gyre dynamics in terms of bottom stress, in order to make a clear exposition of the dynamics. A sink of vorticity is required for the formation of gyres and it is well established that bottom stress can take this role (Stommel 1948). In the same vein, baroclinicity is also omitted from this study. A barotropic system is sufficient to test the hypothesis of Nadeau and Ferrari (2015) and build on the linear ACC theory outlined by Johnson and Hill (1975), Krupitsky and Cane (1994), and Wang and Huang (1995). With this simple barotropic representation, many aspects of Southern Ocean flow are missing and it must be stressed that it is not the intention to provide an exact representation of Southern Ocean dynamics. To make this distinction clear, the barotropic representation of the ACC in the model results will be referred to as a "circumpolar current." The limitations of our reduced system are discussed in section 5 along with some speculation about how the ideas presented in this study might transfer to more complex cases.

The remainder of this paper is structured as follows. Section 2 outlines the methods, presenting the model utilized and all equations of motion relevant to the results of this study. In section 3, results are presented detailing the effect of topographic variation on a barotropic circumpolar current and the zonal momentum budget. Section 4 gives a new insight into the role of topography in the formation of Southern Ocean gyres. Section 5 contains a general discussion of how our results relate to the current literature. A summary is provided in section 6 .

\section{Methods}

\section{a. Governing equations}

For a fluid with uniform density, the hydrostatic Navier-Stokes equations are given by

$$
\rho_{0}\left(\frac{D \mathbf{u}}{D t}+f \mathbf{k} \times \mathbf{u}\right)=-\nabla p-\rho_{0} g \mathbf{k}+\mu \nabla^{2} \mathbf{u},
$$

with conservation of mass

$$
\nabla \cdot \mathbf{u}=0
$$

where $\rho_{0}$ is the reference density; $D \mathbf{u} / D t=\partial \mathbf{u} / \partial t+\mathbf{u} \cdot \nabla \mathbf{u}$ is the material derivative; $\mathbf{u}=\{u, v, w\}$ is the velocity vector, where $u, v$, and $w$ are the velocity components in the $x, y$, and $z$ directions; $f$ is the Coriolis parameter; $\mathbf{k}$ is the unit vector pointing upward; $p$ is the pressure; $\mu$ is the dynamic viscosity coefficient, and the horizontal components of Earth's rotation have been neglected. For convenience, $u$ will be referred to as the eastward velocity and $v$ as the northward velocity.

This study uses three forms of Eq. (1): the barotropic vorticity equation, barotropic potential vorticity equation, and barotropic zonal momentum equation [see Patmore (2018) for derivations]. The merits of barotropic vorticity and potential vorticity are well described in Jackson et al. (2006). These equations are equivalent to each other in a domain with a flat bottom, and it is only when topography is introduced that the benefit of each becomes clear. Potential vorticity and barotropic vorticity are primarily used to understand gyre dynamics. The barotropic zonal momentum equation is useful for understanding the interaction between topography and circumpolar flow.

\section{1) BAROTROPIC VORTICITY EQUATION}

Taking the depth integral of the momentum Eqs. (1) gives the barotropic momentum equations. The vertical component of the curl of the barotropic momentum equations gives the barotropic vorticity equation. The steady barotropic vorticity equation is

$$
\begin{aligned}
& \rho_{0}\left[\mathbf{k} \cdot \nabla \times\left(\bar{H} \overline{\mathbf{u}} \cdot \nabla \overline{\mathbf{u}}+\bar{H} \overline{\overline{\mathbf{u}}^{\prime} \cdot \nabla \mathbf{u}^{\prime}}\right)+\bar{H} \beta \bar{v}\right] \\
& \quad=\mathbf{k} \cdot \nabla \bar{p}_{b} \times \nabla h+\mathbf{k} \cdot \nabla \times\left(\overline{\boldsymbol{\tau}}_{w}-\overline{\boldsymbol{\tau}}_{b}\right)+\mu \bar{H} \nabla^{2} \bar{\zeta},
\end{aligned}
$$

where overbars signify the time mean; primes denote the deviation from the time mean; $H=h+\eta$ is the water column thickness, with $h$ being the distance from the resting ocean surface to the bottom topography and $\eta$ being the SSH anomaly; $\beta=d f / d y ; p_{b}=\rho_{0} g H$ is the bottom pressure; $\boldsymbol{\tau}_{w}$ is the wind stress; $\boldsymbol{\tau}_{b}$ is the bottom stress; and $\zeta=d v / d x-d u / d y$ is the relative vorticity. The term $\mathbf{k} \cdot \nabla p_{b} \times \nabla h$ is referred to as the bottom pressure torque. This is the Reynolds averaged form of the barotropic vorticity equation. Covariance in the constituents of the $H^{\prime}$ terms that result from Reynolds averaging is assumed to be small and is neglected.

The barotropic vorticity equation is useful for decomposing the dynamics of gyres. In a domain with variable topography, the barotropic vorticity equation quantifies the influence of the topography through bottom pressure torque (Holland 1972; Hughes and De Cuevas 2001; Jackson et al. 2006). The primary barotropic vorticity balance over topography is between $\mathbf{k} \cdot \nabla \bar{p}_{b} \times \nabla h$ and $\rho_{0} \bar{H} \beta \bar{v}$ (Holland 1972). The time-mean 
form of the equation provides insight into the influence of eddying effects on the time-mean flow through the Reynolds stress term $\rho_{0} \mathbf{k} \cdot \nabla \times\left(\bar{H} \overline{\mathbf{u}^{\prime} \cdot \nabla \mathbf{u}^{\prime}}\right)$ (Hughes and Ash 2001; Thompson and Richards 2011).

\section{2) BAROTROPIC POTENTIAL VORTICITY EQUATION}

The barotropic potential vorticity equation is obtained by taking the depth average of the vertical component of the curl of the momentum equations. The barotropic potential vorticity equation is

$$
\frac{D}{D t}\left(\frac{f+\zeta}{H}\right)=\frac{1}{\rho_{0} H} \mathbf{k} \cdot \nabla \times\left(\frac{\boldsymbol{\tau}_{w}-\boldsymbol{\tau}_{b}}{H}\right)+\frac{\nu}{H} \nabla^{2} \zeta,
$$

where $\nu=\mu / \rho_{0}$ is the kinematic viscosity coefficient. This equation is a useful alternative to the barotropic vorticity equation for assessing gyre dynamics and highlighting the effects of topographic steering in general. In this study, potential vorticity is reserved solely for theoretical discussion of both gyre and circumpolar current dynamics. As a leading-order approximation, Eq. (4) can be simplified to

$$
D(f / H) / D t=0,
$$

where flow follows contours of $f / H$ so as to conserve potential vorticity; $\zeta$ can be neglected from the lefthand side of Eq. (4) when scales are larger than the Rhines scale $(U / \beta)^{1 / 2}$ (Naveira Garabato et al. 2013). Neglecting $\zeta$ gives the linearized form of potential vorticity. Terms on the right-hand side of the linearized form of Eq. (4) cause flow to deviate from contours of $f / H$ and are important for the formation of gyres. Potential vorticity is therefore a natural context in which to frame gyre formation in a conceptual sense. The Reynolds stress term is associated with $\zeta$, and thus Reynolds averaging is not relevant for the linearized form of potential vorticity.

\section{3) BAROTROPIC ZONAL MOMENTUM EQUATION}

The barotropic zonal momentum equation is the depth and zonal integral of the zonal momentum equation. The steady, Reynolds-averaged barotropic zonal momentum equation is defined as

$$
\rho_{0}\left[\oint \bar{H}\left(\bar{\zeta} \bar{v}+\overline{\zeta^{\prime} v^{\prime}}\right) d x+\oint \frac{\bar{H}}{2} \frac{\partial\left(\bar{u}^{2}+\bar{v}^{2}\right)}{\partial x} d x+\oint \frac{\bar{H}}{2} \frac{\partial\left(u^{\prime 2}+v^{\prime 2}\right)}{\partial x} d x\right]=\oint \bar{p}_{b} \frac{\partial h}{\partial x} d x+\oint \bar{\tau}_{w}^{x} d z-\oint \bar{\tau}_{b}^{x} d x+\mu \oint \bar{H} \nabla^{2} \bar{u} d x
$$

Circular integrals denoted by $\oint d x$ are taken over a zonally re-entrant channel domain. The Coriolis term is absent in Eq. (6) because $\oint H f v d x=0$ in this channel setting.

In the Southern Ocean, the primary source of momentum is wind stress. The key zonal momentum sink balancing the wind stress is form stress, $\oint \bar{p}_{b}(\partial h / \partial x) d x$, which is generated in the presence of topographic variations. The barotropic zonal momentum equation is used in this study to link variations in topography to changes in volume transport.

\section{b. Model}

Idealized modeling experiments are conducted with the Massachusetts Institute of Technology general circulation model (MITgcm; Marshall et al. 1997a,b), a model capable of simulating the full Navier-Stokes equations. Velocities are taken to be uniform in the vertical, achieved by using a grid with one layer. Variable seabed topography is incorporated using partial cells. The density is taken to be constant everywhere.

Table 1 gives a summary of the variations in model configuration for all simulations presented in this study. All simulations are based on a one-layer channel of 5000-m depth, to which a variety of ridge geometries are added. The initial domain has size $L_{x}=7200 \mathrm{~km}$ and $L_{y}=7200 \mathrm{~km}$, where $L_{x}$ and $L_{y}$ represent the length of the domain in the zonal and meridional directions, respectively. All models have a zonally uniform surface wind stress. The initial results have a domain uniform zonal wind stress of magnitude $\tau_{0}=0.144 \mathrm{~N} \mathrm{~m}^{-2}$. The initial results in section 4 with $L_{y}=$ $3600 \mathrm{~km}$ are forced with a "sinusoidal" zonal wind stress that varies in the meridional direction according to

$$
\tau_{w}^{x}=0.5 \tau_{0}\left[1-\cos \left(2 \pi y / L_{y}\right)\right],
$$

where $\tau_{0}=0.144 \mathrm{~N} \mathrm{~m}^{-2}$ is the peak wind stress. In these latter runs, both the wind stress and its curl drop to zero at the domain boundaries to the north and south. The extended domain in section 4 with $L_{y}=7200 \mathrm{~km}$ uses the same wind forcing in the southern half of the domain and $\tau_{w}^{x}=0$ otherwise.

The horizontal grid spacing is $12.5 \mathrm{~km}$. The time step is $1 \mathrm{~h}$. All simulations are run for $10 \mathrm{yr}$. The output is recorded at intervals of $24 \mathrm{~h}$. All results are averaged over the outputs from the final simulation year, when the model is statistically steady. The model uses the $\beta$-plane approximation, with the Coriolis parameter $f=f_{0}+\beta y$, where $f_{0}=2 \omega \sin \phi_{0}, \beta=\left(2 \omega \cos \phi_{0}\right) / a$, 
TABLE 1. List of all model configurations. The columns represent model identification number (ID), zonal length of domain, meridional width of domain, existence of central meridional wall ( $\mathrm{N}=$ no; $\mathrm{Y}=$ yes); height of submarine topography, width of submarine topography, "curl" indicating sinusoidal wind forcing and "uniform" for uniform surface wind forcing, and prescribed bottom stress coefficient.

\begin{tabular}{|c|c|c|c|c|c|c|c|}
\hline ID & $L_{x}(\mathrm{~km})$ & $L_{y}(\mathrm{~km})$ & Wall & Ridge height (m) & Ridge width (km) & Wind & $C_{d}$ \\
\hline 01 & 7200 & 7200 & $\mathrm{~N}$ & 2000 & 500 & Uniform & 0.0025 \\
\hline 02 & 7200 & 7200 & $\mathrm{~N}$ & 2000 & 1000 & Uniform & 0.0025 \\
\hline 03 & 7200 & 7200 & $\mathrm{~N}$ & 2000 & 1500 & Uniform & 0.0025 \\
\hline 04 & 7200 & 7200 & $\mathrm{~N}$ & 2000 & 2000 & Uniform & 0.0025 \\
\hline 05 & 7200 & 7200 & $\mathrm{~N}$ & 2000 & 2500 & Uniform & 0.0025 \\
\hline 06 & 7200 & 7200 & $\mathrm{~N}$ & 2000 & 3000 & Uniform & 0.0025 \\
\hline 07 & 7200 & 7200 & $\mathrm{~N}$ & 2000 & 3500 & Uniform & 0.0025 \\
\hline 08 & 7200 & 7200 & $\mathrm{~N}$ & 2000 & 4000 & Uniform & 0.0025 \\
\hline 09 & 7200 & 7200 & $\mathrm{~N}$ & 2000 & 1000 & Uniform & 0.0050 \\
\hline 10 & 7200 & 7200 & $\mathrm{~N}$ & 2000 & 1000 & Uniform & 0.0075 \\
\hline 11 & 7200 & 7200 & $\mathrm{~N}$ & 2000 & 2000 & Uniform & 0.0050 \\
\hline 12 & 7200 & 7200 & $\mathrm{~N}$ & 2000 & 2000 & Uniform & 0.0075 \\
\hline 13 & 7200 & 7200 & $\mathrm{~N}$ & 2000 & 4000 & Uniform & 0.0050 \\
\hline 14 & 7200 & 7200 & $\mathrm{~N}$ & 2000 & 4000 & Uniform & 0.0075 \\
\hline 15 & 7200 & 3600 & $\mathrm{~N}$ & 0 & 1000 & Curl & 0.0025 \\
\hline 16 & 7200 & 3600 & $\mathrm{~N}$ & 500 & 1000 & Curl & 0.0025 \\
\hline 17 & 7200 & 3600 & $\mathrm{~N}$ & 1000 & 1000 & Curl & 0.0025 \\
\hline 18 & 7200 & 3600 & $\mathrm{~N}$ & 2000 & 1000 & Curl & 0.0025 \\
\hline 19 & 7200 & 3600 & $\mathrm{Y}$ & 2000 & 1000 & Curl & 0.0025 \\
\hline 20 & 7200 & 3600 & $\mathrm{~N}$ & 2000 & 2000 & Curl & 0.0025 \\
\hline 21 & 7200 & 3600 & $\mathrm{~N}$ & 2000 & 4000 & Curl & 0.0025 \\
\hline 22 & 7200 & 7200 & $\mathrm{~N}$ & 2000 & 1000 & Curl & 0.0025 \\
\hline 23 & 7200 & 7200 & $\mathrm{~N}$ & 2000 & 2000 & Curl & 0.0025 \\
\hline 24 & 7200 & 7200 & $\mathrm{~N}$ & 2000 & 4000 & Curl & 0.0025 \\
\hline 25 & 7200 & 7200 & $\mathrm{~N}$ & 2000 & 1000 & Curl & 0.0050 \\
\hline 26 & 7200 & 7200 & $\mathrm{~N}$ & 2000 & 2000 & Curl & 0.0050 \\
\hline 27 & 7200 & 7200 & $\mathrm{~N}$ & 2000 & 4000 & Curl & 0.0050 \\
\hline
\end{tabular}

$\omega=7.2921 \times 10^{-5} \mathrm{~s}^{-1}$ is the angular velocity of Earth, $\phi_{0}$ is a fixed latitude, and $a=6371 \mathrm{~km}$ is Earth's radius. In all model simulations the southern latitudinal boundary of the model is set to $\phi_{0}=-60.85^{\circ}$ such that $f_{0}=-1.27393 \times 10^{-4} \mathrm{~s}^{-1}$ and $\beta=1.1144 \times$ $10^{-11} \mathrm{~m}^{-1} \mathrm{~s}^{-1}$. The model uses a constant reference density $\rho_{0}$. The hydrostatic approximation means that $p=\rho_{0} g(\eta-z)$, where $g=9.8 \mathrm{~m} \mathrm{~s}^{-2}$. All solid lateral boundaries have a free-slip condition. The upper boundary is governed by a nonlinear free surface, the lower boundary is governed by a quadratic drag with drag coefficient of $C_{d}=2.5 \times 10^{-3}$, and there is a constant kinematic viscosity coefficient of $\nu=10 \mathrm{~m}^{2} \mathrm{~s}^{-1}$.

\section{Topographic control of the Antarctic Circumpolar Current}

Previous studies have shown that topography can regulate the volume transport of the ACC (Munk and Palmén 1951; Wolff et al. 1991; Tansley and Marshall 2001; Nadeau and Ferrari 2015). The mechanism for this is form stress balancing wind stress in the zonal momentum balance. Form stress can only exist where there is topographic variation, and without it the volume transport of the ACC would be orders of magnitude larger than observed (Munk and Palmén 1951). Relationships between seabed ridge width and zonal volume transport are well established for the linear case for regions of both blocked and unblocked geostrophic contours (Krupitsky and Cane 1994; Wang and Huang 1995). Linear dynamics are significantly distinct from the nonlinear counterpart due to the absence of topographic Rossby waves (Wang and Huang 1995). The effects of geometric variations are yet to be explored in the nonlinear setting with unblocked geostrophic contours. This section considers the role of ridge width on the dynamics of a barotropic circumpolar current in this setting and investigates the dynamical details of the relationships that emerge.

\section{a. The relationship between ridge width and channel flow dynamics}

To assess the impact of topography on a circumpolar current, an idealized experiment is conducted where ridge width is varied. The simulations in this experiment are forced with a uniform zonal wind over the entire domain in order to simplify the dynamics. Figures $2 \mathrm{a}-\mathrm{c}$ shows the barotropic streamfunction for 

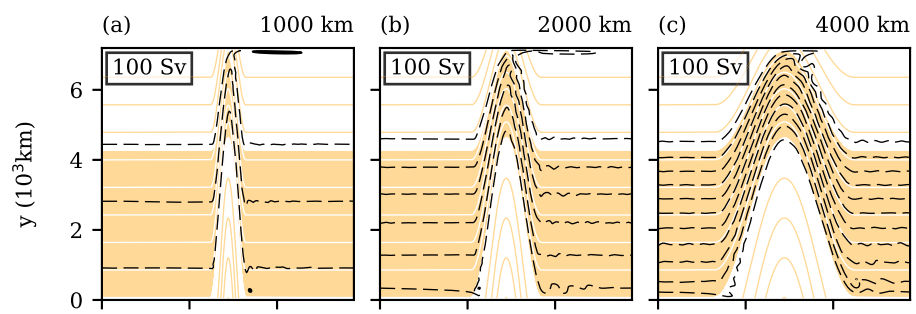

Wind Stress
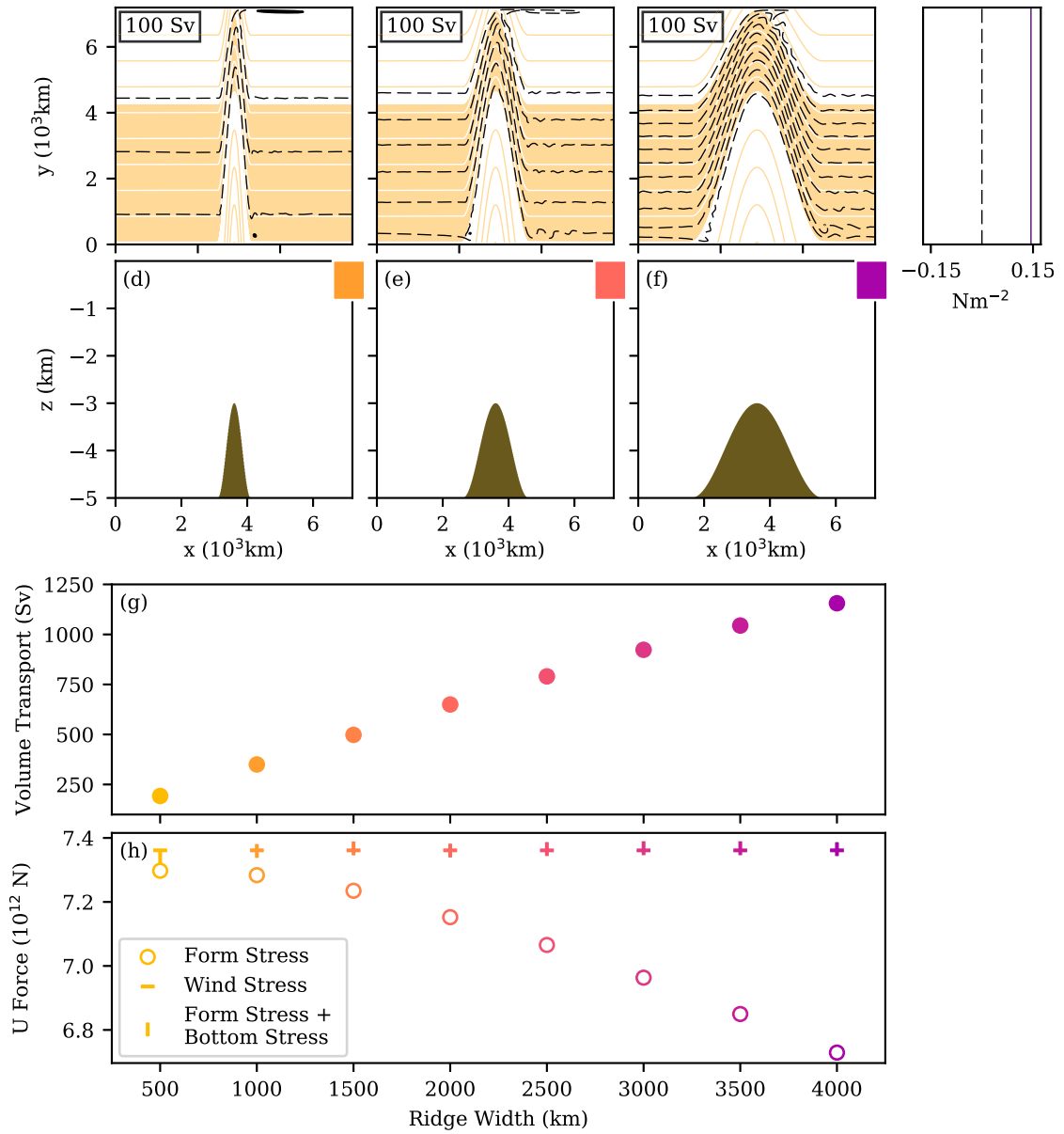

FIG. 2. Results for ridge width variations with uniform wind forcing for models $01-08$. The barotropic streamfunction for three ridge widths: (a) $1000 \mathrm{~km}$ (model 02), (b) $2000 \mathrm{~km}$ (model 04), and (c) $4000 \mathrm{~km}$ (model 08). Black lines are streamlines. Beige/white lines represent geostrophic $(f / h)$ contours. The beige fill represents any region of unblocked geostrophic contours, defined by contours that do not intersect with the model walls to the north or south. The boxes in the top-left corner of (a)-(c) signify the contour spacing of the streamfunction. (d)-(f) The topographic profiles of (a)-(c), respectively. The colored boxes to the top-right of (d)-(f) are associated with ridge width. This color association is used in other figures in this paper. (g) Net zonal volume transport against ridge width for models 01-08. (h) Domain integral of terms in the zonal momentum budget for varying ridge width for models $01-08$. The momentum terms shown are the negative of the form stress $\left(-\int \bar{p}_{b} \partial h / \partial x d A\right)$, the wind stress $\left(\int \bar{\tau}_{w}^{x} d A\right)$, and the sum of the negative of the form stress and the bottom stress $\left(-\int \bar{p}_{b} \partial h / \partial x+\bar{\tau}_{b}^{x} d A\right)$.

simulations 02,04 , and 08 with varying ridge widths $(1000$, 2000 , and $4000 \mathrm{~km})$. Unlike experiments that vary ridge height (Krupitsky and Cane 1994; Wang and Huang 1995; Krupitsky et al. 1996; Nadeau and Ferrari 2015), varying ridge width has no effect on the range of blocked $f / H$ here. The results show deviation of flow over topography due to conservation of potential vorticity, demonstrating the leading-order role of Eq. (5). The equatorward deflection of geostrophic contours causes a proportion of them to become blocked through intersection with the solid domain boundaries to the north and south. Areas of unblocked geostrophic contours are signified in Figs. 2a-c by the shaded region. Flow approximately follows contours of $f / H$ and is confined to this shaded area. Flow cannot intersect with a solid boundary and little flow develops in the unshaded region. The results in Figs. 2a-c show that the volume transport increases as the ridge width increases. This is confirmed by results in Fig. $2 \mathrm{~g}$, which shows the volume transport for a larger set of ridge width variations. The barotropic zonal momentum budget has been calculated for the same set of experiments, with the quantities shown in Fig. $2 \mathrm{~h}$. The momentum budget 
in Fig. $2 \mathrm{~h}$ confirms that the primary balance is between form stress and wind stress (Munk and Palmén 1951; Wolff et al. 1991; Stevens and Ivchenko 1997; Masich et al. 2015).

For all simulations, there is a small proportion of the wind stress that is balanced by bottom stress, which increases with ridge width (Fig. $2 \mathrm{~h}$ ). As ridge width increases there is a small decrease in the momentum sink via form stress. Bottom stress accounts for the reduction, increasing with increased ridge width. The relationship outlined is a result of the bottom stress term in the zonal momentum budget being a function of zonal velocities. As the volume transport through the channel increases there is an increase in the zonal velocities resulting in a larger bottom stress momentum sink.

\section{b. Form stress}

Flow over topography results in the generation of form stress. While form stress is well known in the literature, the concept is integral to our analysis and as a result, we provide a detailed background of this term.

In Eq. (6), the term associated with form stress is $\oint \bar{p}_{b}(\partial h / \partial x) d x$. Since $\bar{p}_{b}=\rho_{0} g(\bar{\eta}+h)$ under the hydrostatic approximation, and $\oint h(\partial h / \partial x) d x=0$, form stress can be rewritten as

$$
\begin{aligned}
\oint \bar{p}_{b} \frac{\partial h}{\partial x} d x & =\rho_{0} g \oint(\bar{\eta}+h) \frac{\partial h}{\partial x} d x \\
& =\rho_{0} g \oint \bar{\eta} \frac{\partial h}{\partial x} d x,
\end{aligned}
$$

where $\eta$ is defined as before: the anomaly in SSH.

Form stress represents a pressure difference across a topographic feature, which is associated with meridional flow induced by topographic steering due to conservation of potential vorticity. In the Southern Hemisphere, SSH increases toward the left of a largescale geostrophic flow. The equatorward flow on the western flank of a ridge and poleward flow on the eastern flank results in a SSH dip toward the ridge crest, illustrated in the conceptual schematic shown in Fig. 3a. Under the assumption of a symmetric linear topographic profile, form stress would be

$$
\begin{aligned}
\rho_{0} g \oint \bar{\eta} \frac{\partial h}{\partial x} d x & =\rho_{0} g \int_{x_{0}}^{x_{2}} \bar{\eta} \frac{\partial h}{\partial x} d x \\
& =\rho_{0} g\left(\int_{x_{0}}^{x_{1}} \bar{\eta} \frac{\partial h}{\partial x} d x+\int_{x_{1}}^{x_{2}} \bar{\eta} \frac{\partial h}{\partial x} d x\right) \\
& =\rho_{0} g\left|\frac{\partial h}{\partial x}\right|\left(\int_{x_{1}}^{x_{2}} \bar{\eta} d x-\int_{x_{0}}^{x_{1}} \bar{\eta} d x\right),
\end{aligned}
$$

where $x_{0}$ represents the leading ridge base, $x_{1}$ is the ridge peak, and $x_{2}$ is the trailing ridge base. If the dip (a)

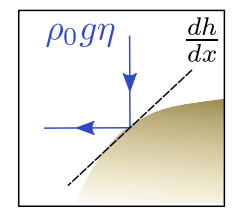

(b)

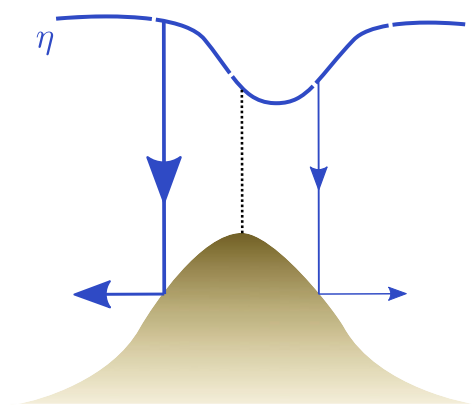

$\eta$

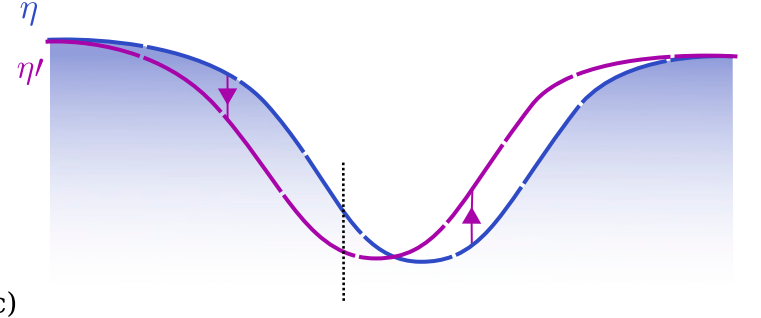

(c)

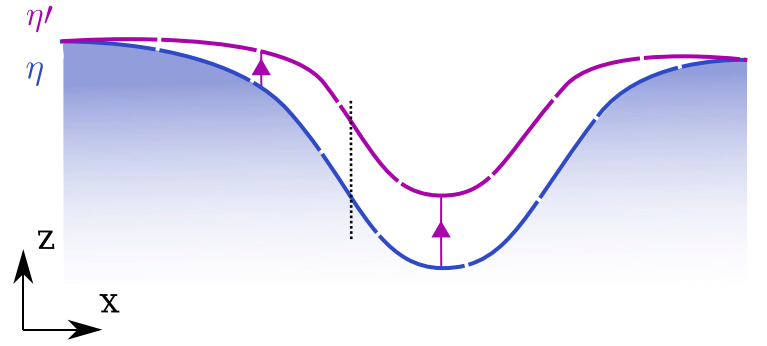

FIG. 3. (a) Schematic representation of form stress. A lateral offset in the sea surface causes the water column to be thicker on the western ridge flank than on the eastern flank. The difference in water column thickness is associated with a pressure difference across the ridge. The downward arrows signify that the pressure is larger on the western ridge flank than the eastern flank, leading to a net westward force, which is associated with form stress. Also shown are mechanisms for changes in form stress arising from adjustments in SSH: (b) a form stress reduction through a reduced lateral offset in SSH with respect to the topographic ridge and (c) a form stress reduction via a reduced dip in SSH. The vertical line in (b) and (c) represents the location of the ridge peak.

in the sea surface lies symmetrically over the topography $\int_{x_{1}}^{x_{2}} \bar{\eta} d x=\int_{x_{0}}^{x_{1}} \bar{\eta} d x$ and there would be no form stress. The conditions for form stress to exist are encapsulated by the fact that $\oint \bar{\eta}(\partial h / \partial x) d x \neq 0$ if and only if there is a zonal offset of the topography and the anomaly in SSH. A zonal offset results in a thicker water column over the western ridge flank versus the eastern flank and hence a difference in pressure across the ridge.

\section{c. The source of the lateral offset}

The lateral offset in the SSH anomaly is associated with a vorticity sink perturbing flow from geostrophic contours, which can result from the presence of either bottom stress or inertia (Naveira Garabato et al. 2013). 
Where there is an exact balance in Eq. (3) between the advection of planetary vorticity $\left(-\rho_{0} \bar{H} \beta \bar{v}\right)$ and the bottom pressure torque $\left(\mathbf{k} \cdot \nabla \bar{p}_{b} \times \nabla h\right)$, flow is aligned with geostrophic contours (Jackson et al. 2006). Where this balance is imperfect, flow deviates from geostrophic contours. The vorticity contributions of bottom stress and inertia are linked to an asymmetric deviation of flow relative to geostrophic contours as it traverses topography.

Bottom stress curl associated with the circumpolar jet is anticyclonic to the left of the jet center and cyclonic to the right. On the downstream ridge flank, the bottom stress curl works in concert with bottom pressure torque to the left and in opposition to the right of the jet center, which is associated with a downslope deviation of the flow (Jackson et al. 2006). The meridionally uniform topography reduces the bottom pressure torque to $-\left(d \bar{p}_{b} / d y\right)(d h / d x)$, with a change in sign across the ridge. As a result, the relationship with bottom stress curl is reversed and on the upstream ridge flank flow deviates upslope.

The role of inertia is similar to that of bottom stress, but differences occur in the associated downstream dynamics. The existence of stationary topographic Rossby waves causes flow to oscillate about geostrophic contours eastward of the ridge crest (McCartney 1976). The overall downstream deviation is then a linear combination of the downslope deflection (as described for the bottom stress case) and Rossby wave oscillation (McCartney 1976).

The asymmetry in a linear case with blocked geostrophic contours is achieved through bottom stress curl only (Krupitsky and Cane 1994; Wang and Huang 1995). The blocked geostrophic contours generate a lateral boundary layer, which is dynamically similar to a Stommel gyre western boundary current (Stommel 1948). The flow crosses geostrophic contours via the presence of bottom stress curl and an asymmetry develops across the topography.

The asymmetric deviation of flow due to either bottom stress or inertia is associated with an SSH anomaly which is displaced eastward, as depicted in Fig. 3. The offset of the SSH profile generates a pressure difference across the topography, creating the form stress and a momentum sink. In the nonlinear unblocked case explored in this section, the lateral offset occurs in association with both Rossby waves and bottom stress curl.

\section{d. Mechanisms for perturbing form stress}

It has been shown by Fig. $2 \mathrm{~h}$ that for changing topography, form stress remains the primary momentum sink for the wind stress. The wind stress remains constant between simulations, and so must the form stress. Despite form stress remaining approximately constant, it has two varying components between simulations, the ridge width $d x$ and SSH $\eta$. Although $d x$ varies, it has no net effect on the form stress because changes in the two $d x$ terms of Eq. (7) cancel as the bounds of integration change. $\eta$ on the other hand is determined by the dynamics and form stress is strongly dependent on this term. How $\eta$ evolves in response to changes in topography is integral to the relationships that we observe.

Figure 3 illustrates two ways in which the sea surface influences form stress. The first mechanism, shown in Fig. 3b, comprises a reduction in the lateral offset between the SSH profile and the topography. The dip in the sea surface shifts westward. The sea surface rises to the east of the ridge peak and sinks to the west of the ridge peak. As a result, $\int_{x_{1}}^{x_{2}} \eta d x$ decreases in magnitude and $\int_{x_{0}}^{x_{1}} \eta d x$ increases in magnitude. This causes a reduction in the magnitude of $\int_{x_{1}}^{x_{2}} \eta d x-\int_{x_{0}}^{x_{1}} \eta d x$ and through Eq. (8) from stress is reduced. The second, illustrated in Fig. 3c, constitutes a reduction in the sea surface dip induced by the meridional velocities over the ridge. A change in form stress due an adjustment in the dip of sea surface is well illustrated by Stewart and Hogg (2017) and the details of this effect can be seen by reforming the form stress term. Following LaCasce and Isachsen (2010), integration by parts gives

$$
\rho_{0} g \oint \bar{\eta} \frac{\partial h}{\partial x} d x=-\rho_{0} g \oint h \frac{\partial \bar{\eta}}{\partial x} d x=-\rho_{0} g \oint f h v_{g} d x,
$$

where $v_{g}$ is the geostrophic meridional velocity. Any changes in the sea surface dip are associated with changes in $\partial \eta / \partial x$ and hence the meridional velocities. Thus, by Eq. (9) a larger dip (or $\partial \eta / \partial x$ ) is associated with a larger form stress.

\section{$e$. The role of form stress in regulating volume transport}

The relationship between volume transport and ridge width shown in Fig. 2 can be explained in terms of form stress. Figure 4 a shows the profiles of $\eta$ over the topography as the ridge width is varied. As the ridge width is decreased, the sea surface dip reduces. There is a simultaneous shift in the profile to the east, which is observed in Fig. 4b as an eastward shift in the minimum point of the sea surface profile. These changes have opposing effects on the form stress. The eastward shift causes an increase in form stress and the decreased dip causes a decrease in form stress. The opposing effects act to maintain form stress as the primary balance form the wind stress. The shift must have a slightly larger effect than the dip in order to create the overall marginal increase in form stress shown in Fig. 2h.

Figure $4 \mathrm{c}$ shows the depth and meridionally integrated meridional velocities over the ridge, where the $x$ axis is scaled by ridge width. The meridional velocities over the topography are similar for all ridge widths. They are 
(a)

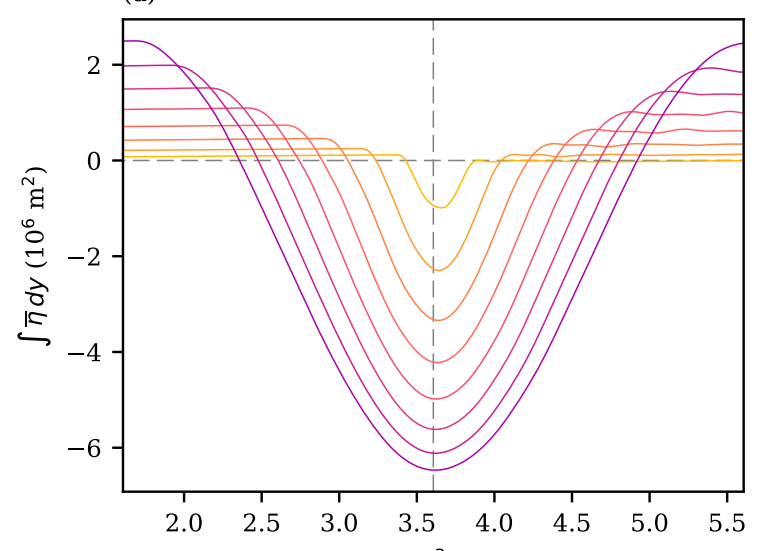

(c) (b)

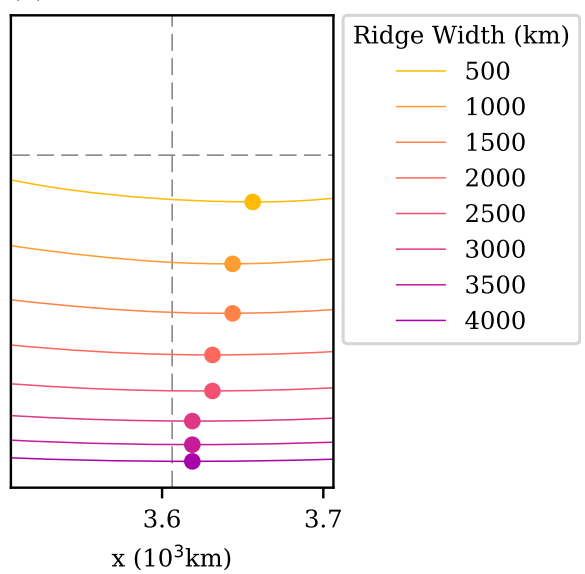

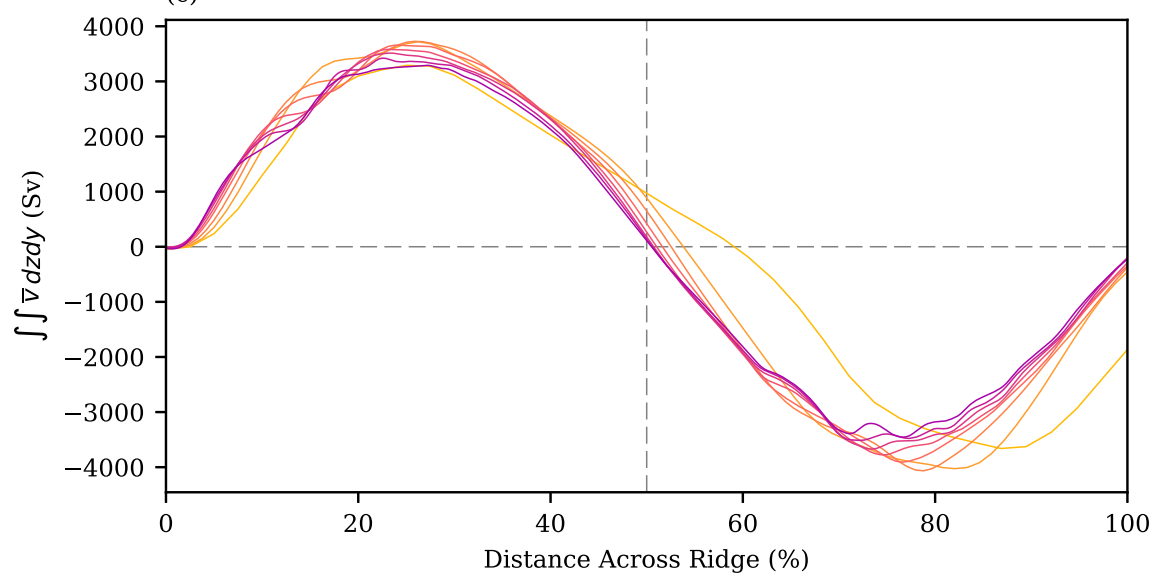

FIG. 4. Meridionally integrated quantities for varying ridge width (models 01-08). (a) Meridionally integrated SSH. (b) Meridionally integrated SSH near the ridge peak. The dots represent the minima of the curves. (c) Meridionally integrated meridional velocities. True distances are given for the $x$ axes in (a) and (b), whereas in (c), the $x$ axis is scaled to the ridge width of each simulation.

linked directly to form stress via Eq. (9) and are sustained by the topographic controls described above.

The relationship between volume transport and ridge width results from the insensitivity of the meridional velocities shown in Fig. 4c. The meridional velocities are approximately constant between simulations (Fig. 4c), while zonal velocities increase with ridge width (not shown). As ridge width increases, the geostrophic contours become more zonal. The flow primarily aligns with geostrophic contours and in order for meridional velocities to remain the same the flow speed must increase, leading to elevated zonal velocities. Higher zonal velocities cause an increase in the volume transport. An equivalent description is as follows. For $U=\int_{-h}^{\eta} u d z$ and $V=\int_{-h}^{\eta} v d z$,

$$
\frac{U}{V} \approx \frac{w}{2 L_{\beta}},
$$

where $w$ is the ridge width and $L_{\beta}$ is the meridional deviation of an $f / H$ contour between the base and peak of the ridge. An approximation of volume transport is found by taking a meridional integral of Eq. (10),

$$
T=\int_{0}^{L_{y}} U d y \approx \frac{w}{2 L_{\beta}} \int_{0}^{L_{y}} V d y,
$$

where $T$ represents the volume transport through the channel. The relationship provided by Eq. (11) shows ridge geometry links the meridional velocities to the volume transport. A constant $\int_{0}^{L_{y}} V d y$ and decreasing $w$ results in a lowered volume transport. This means that the changing circumpolar transport in these simulations can be understood as consequence of the constant meridional velocity magnitudes over the ridge.

In summary, this section has described why a steeper ridge is associated with reduced circumpolar transport. The full mechanism is as follows:

1) Form stress remains approximately constant, matching wind stress. 
2) As ridge width increases, the zonal offset in the SSH dip relative to the ridge decreases. If unopposed, this would reduce form stress.

3) An increasing SSH dip opposes the zonal offset, acting to maintain the form stress.

4) This increasing dip is associated with constant zonal SSH gradients, associated with meridional velocities that are independent of ridge width.

5) Wider ridges alter the geostrophic contours such that the unvarying meridional velocities require an increased zonal volume transport.

\section{f. Dynamical sensitivity to changes in bottom stress}

The momentum sink provided by bottom stress is small in comparison to form stress (Fig. 2h). Despite this, it can be important to the dynamics in less direct ways. The presence of form stress is reliant on bottom stress and/or inertia to generate an offset in the SSH anomaly from the topography (Naveira Garabato et al. 2013). The indirect role that bottom stress has in generating form stress highlights the potential importance of this term to the dynamical system presented. As such, additional simulations are carried out where the bottom stress coefficient is varied (Fig. 5) and we discuss the associated dynamics of this term.

Figure 5a shows that there is a reduction in volume transport in response to an increased bottom stress coefficient, which requires reduced velocities to maintain the bottom stress momentum sink. However, this response cannot retain the exact same momentum balance and a slight adjustment occurs. Figure $5 \mathrm{~b}$ shows that the proportion of the wind stress that is balanced by the bottom stress increases as the bottom stress coefficient increases. A larger reduction in volume transport would be required to maintain the bottom stress momentum sink at its previous level.

Figure $5 \mathrm{~b}$ shows that the increase in the bottom stress momentum sink is balanced by a decrease in form stress. Any change in form stress can be observed in the sea surface profile. Figures $5 \mathrm{c}$ and $5 \mathrm{~d}$ show the SSH profile for the simulations where the bottom stress coefficient is varied. The decrease in form stress is primarily associated with reduction in sea surface dip (Fig. 5c) and there is little change in the sea surface offset (Fig. 5d).

The results show that the relative importance of form stress in the zonal momentum budget reduces with increased bottom stress coefficient. An increase in the bottom stress coefficient causes a decreased volume transport, reducing the sea surface dip and form stress reduces as a result. The volume transport reduction is not sufficient to maintain the bottom stress momentum sink at the same level. Bottom stress increases, balancing the reduction of the form stress momentum sink.

This relationship between volume transport and bottom stress coefficient is contrary to existing results and it is often found that increasing the bottom stress coefficient increases the net zonal volume transport (Tansley and Marshall 2001; Nadeau and Straub 2012; Nadeau and Ferrari 2015; Marshall et al. 2017; Constantinou 2018). The reversed relationship in many of the existing results relies on the presence of a baroclinicity that gives rise to the vertical structure described by Straub (1993). The reversed relationship can also occur in a barotropic setting if the forcing is strong and the bottom stress coefficient is below a particular threshold (Constantinou 2018).

\section{Topographic control of Southern Ocean gyres}

The above sections have shown that the geometry of topography has a significant influence on the volume transport through a channel which is representative of the Southern Ocean. The investigation is now directed toward the role of topography on Southern Ocean gyres. Gyre formation requires a curl in the wind stress. The simulations discussed above were forced with a uniform wind stress and hence gyres did not form; the following simulations are forced with a wind stress with nonzero curl.

\section{a. The dynamics of gyres in regions of blocked geostrophic contours}

The established knowledge of gyre formation in the Southern Ocean indicates a requirement for blocking of geostrophic contours (LaCasce and Isachsen 2010; Nadeau and Ferrari 2015; Munday et al. 2015). In early modeling studies, gyres were formed through the intersection of geostrophic contours with a meridional wall which extends to the surface (LaCasce and Isachsen 2010). However, circulations such as the Ross Gyre occur in absence of any meridional wall. Simulations have been presented where gyres form without the need for a meridional boundary (Krupitsky and Cane 1994; Wang and Huang 1995; Krupitsky et al. 1996; Nadeau and Ferrari 2015; Munday et al. 2015) but these results still rely on blocked geostrophic contours. In these domains, absent of a meridional wall, submarine topography introduces an intersection of geostrophic contours with the northern and southern boundaries of a channel domain. An example of this kind of gyre formation is given in Fig. 6. In a channel domain without topography (Figs. 6a,b), geostrophic contours are strictly zonal and do not intersect with any boundary. A linear free mode 

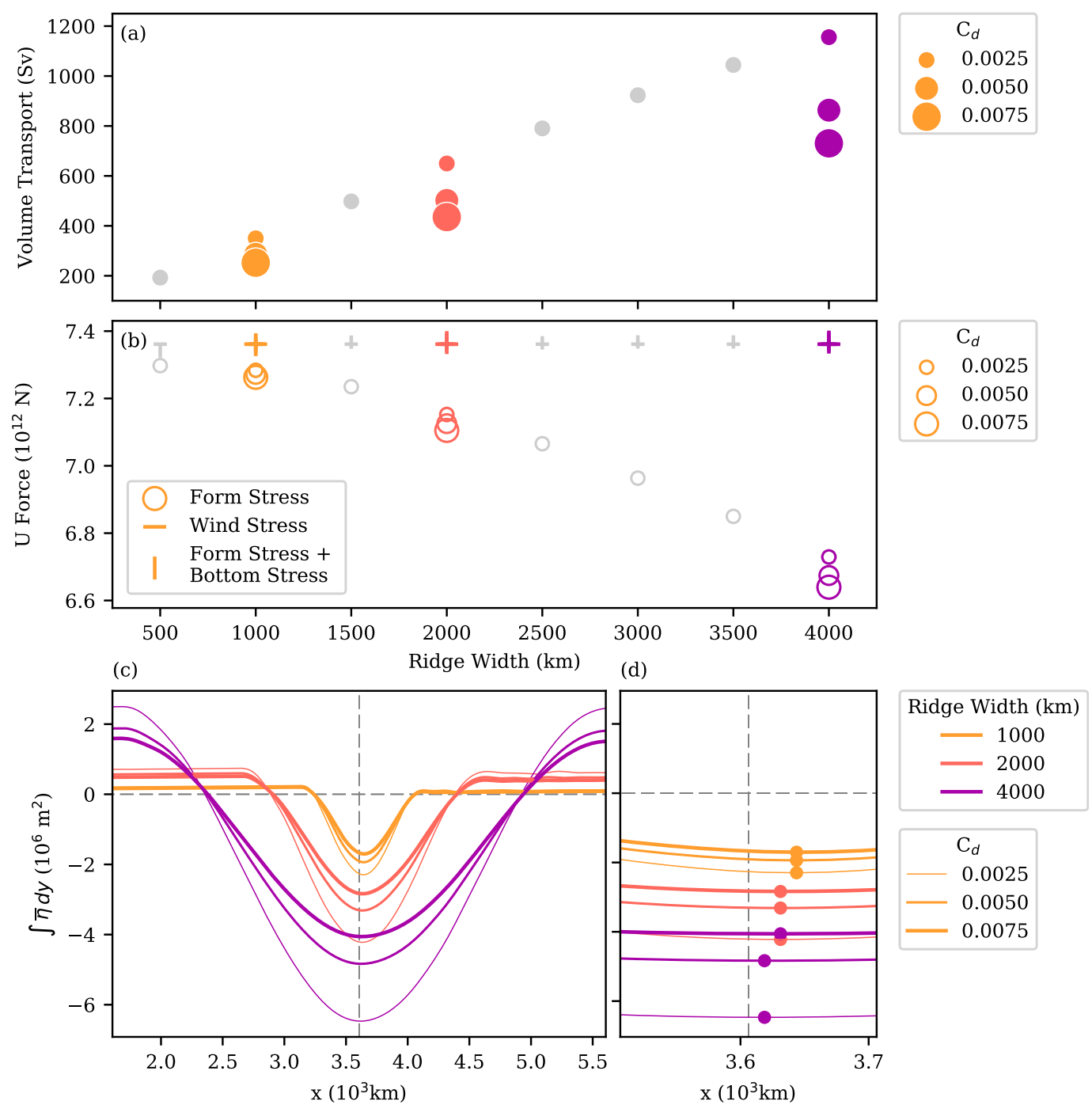

FIG. 5. (a) Net zonal volume transport against ridge width for varying bottom stress coefficient (models 02 , 04, 08, and 09-14). Gray dots represent data shown in Fig. 2. (b) Domain integral of terms in the zonal momentum budget for varying ridge width and bottom stress coefficient. The momentum terms shown are: the negative of the form stress $\left(-\int \bar{p}_{b} \partial h / \partial x d A\right)$, the wind stress $\left(\int \bar{\tau}_{w}^{x} d A\right)$, and the sum of the negative of the form stress and the bottom stress $\left(-\int \bar{p}_{b} \partial h / \partial x+\bar{\tau}_{b}^{x} d A\right)$. (c) Meridionally integrated SSH for varying ridge width and bottom stress coefficient. (d) Meridionally integrated SSH near the ridge peak. The dots represent the minima of the curves.

develops and flow aligns with the zonal geostrophic contours, producing a zonal flow with an enormous volume transport. Topography reduces the volume transport in this situation (Wolff et al. 1991; Tansley and Marshall 2001). As topography is introduced, geostrophic contours become blocked by the domain's northern and southern walls (Figs. 6c,d). Topographic Sverdrup balance ensues and gyres form in the region of blocked geostrophic contours (Krupitsky et al. 1996). Once all geostrophic contours intersect with the boundaries (Figs. 6g,h) there is virtually no circumpolar flow $(\approx 2 \mathrm{~Sv})$. As a result, topographic Sverdrup balance dominates and the system is very similar to a walled domain representative of a midlatitude gyre (Figs. 6i,j).

The pathway for gyre formation through blocked geostrophic contours to the north and south outlined via Fig. 6 is not representative of circulations such as the Ross Gyre. In the Pacific sector of the Southern Ocean, where the Ross Gyre forms, no northern boundary exists with which geostrophic contours can intersect. The question of how Southern Ocean gyres form therefore remains unanswered. Contrary to many existing ideas, the geostrophic contours in Fig. 1 suggest 
(a)

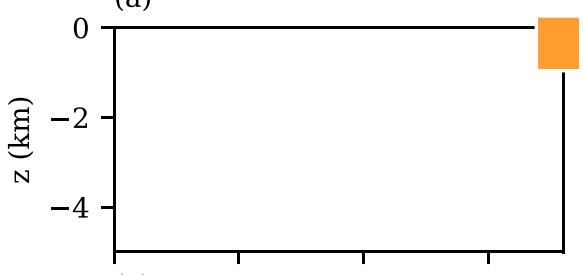

(c)

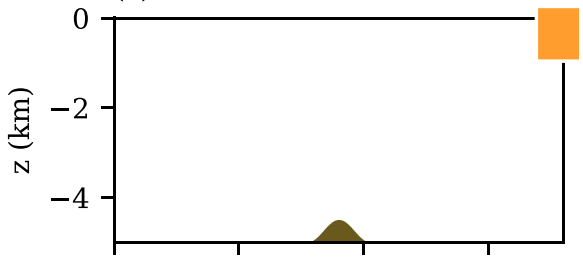

(e)

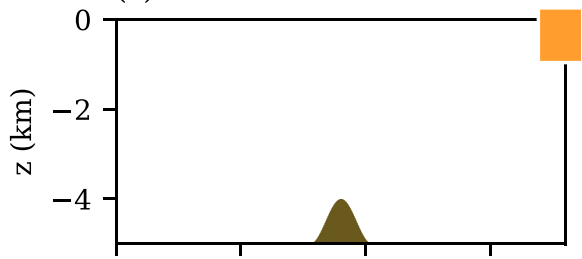

(g)

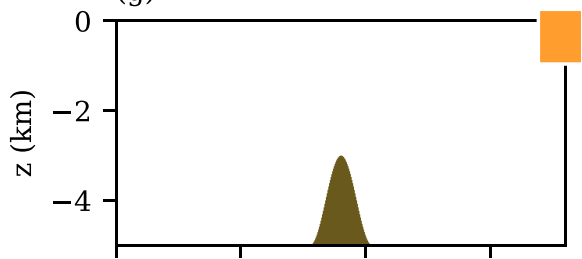

(i)

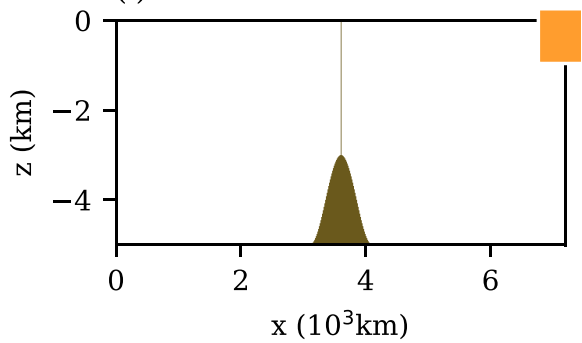

(b)

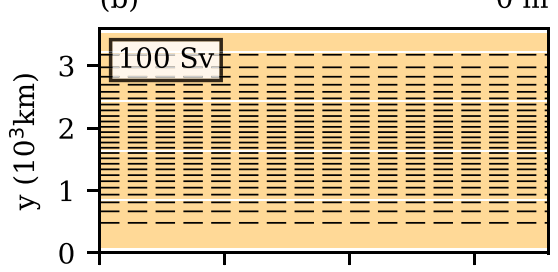

(d)

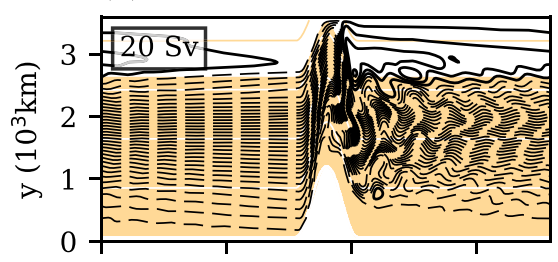

(f)

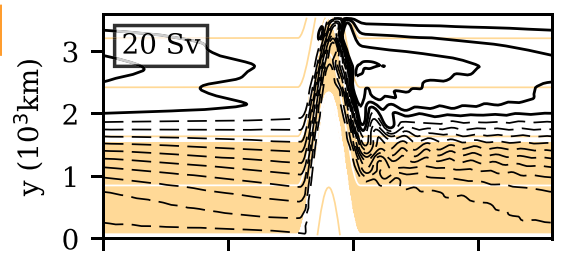

(h)

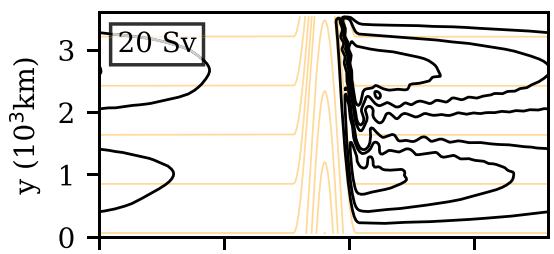

(j)

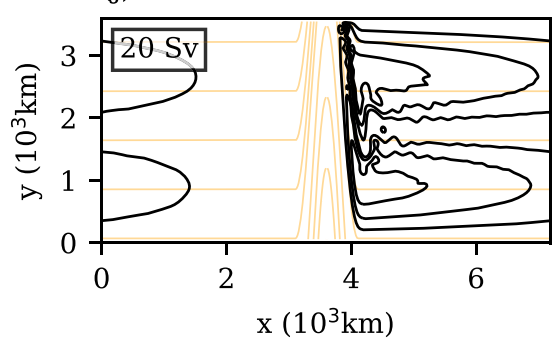

FIG. 6. (right) Plan view of the barotropic streamfunction for channel simulations with a meridional ridge topography varying in height (models 15-19). (left) Side profiles of the topography. The ridge heights are (a),(b) $0 \mathrm{~m}$ (model 15), (c),(d) $500 \mathrm{~m}$ (model 16), (e),(f) $1000 \mathrm{~m}$ (model 17), (g),(h) $2000 \mathrm{~m}$ (model 18), and (i),(j) $2000 \mathrm{~m}$ (model 19); (i) and (j) have a meridional wall over the ridge peak reaching the ocean surface. The colored boxes in the top-right corner of (a), (c), (e), (g), and (i) are associated with ridge width. For coloring and contouring see Fig. 2. The broken streamlines represent circumpolar flow, and the solid streamlines represent stationary eddies or gyre flow.

that gyres can form in regions of unblocked geostrophic contours.

Nadeau and Ferrari (2015) hypothesize that gyre formation in this context is determined by gradients in potential vorticity. However, the effect of increasing gradients in potential vorticity is not fully isolated by Nadeau and Ferrari (2015) because gyre formation is shown to occur through increasing topographic ridge height. Setting up the experiment in this way not only varies gradients in potential vorticity but also the range of blocked geostrophic contours. As highlighted in Fig. 6, blocked geostrophic contours are known to generate gyres, therefore the relationship to potential vorticity gradients in the results of Nadeau and Ferrari (2015) are unclear. We attempt to confirm the hypothesis of Nadeau and Ferrari (2015) here by 

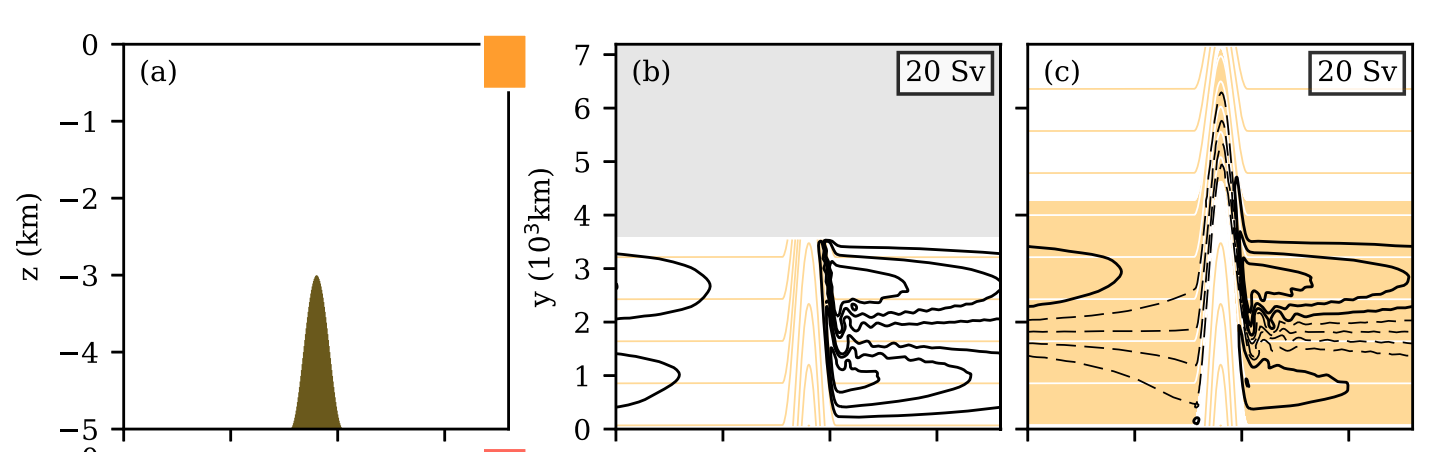

Wind Stress
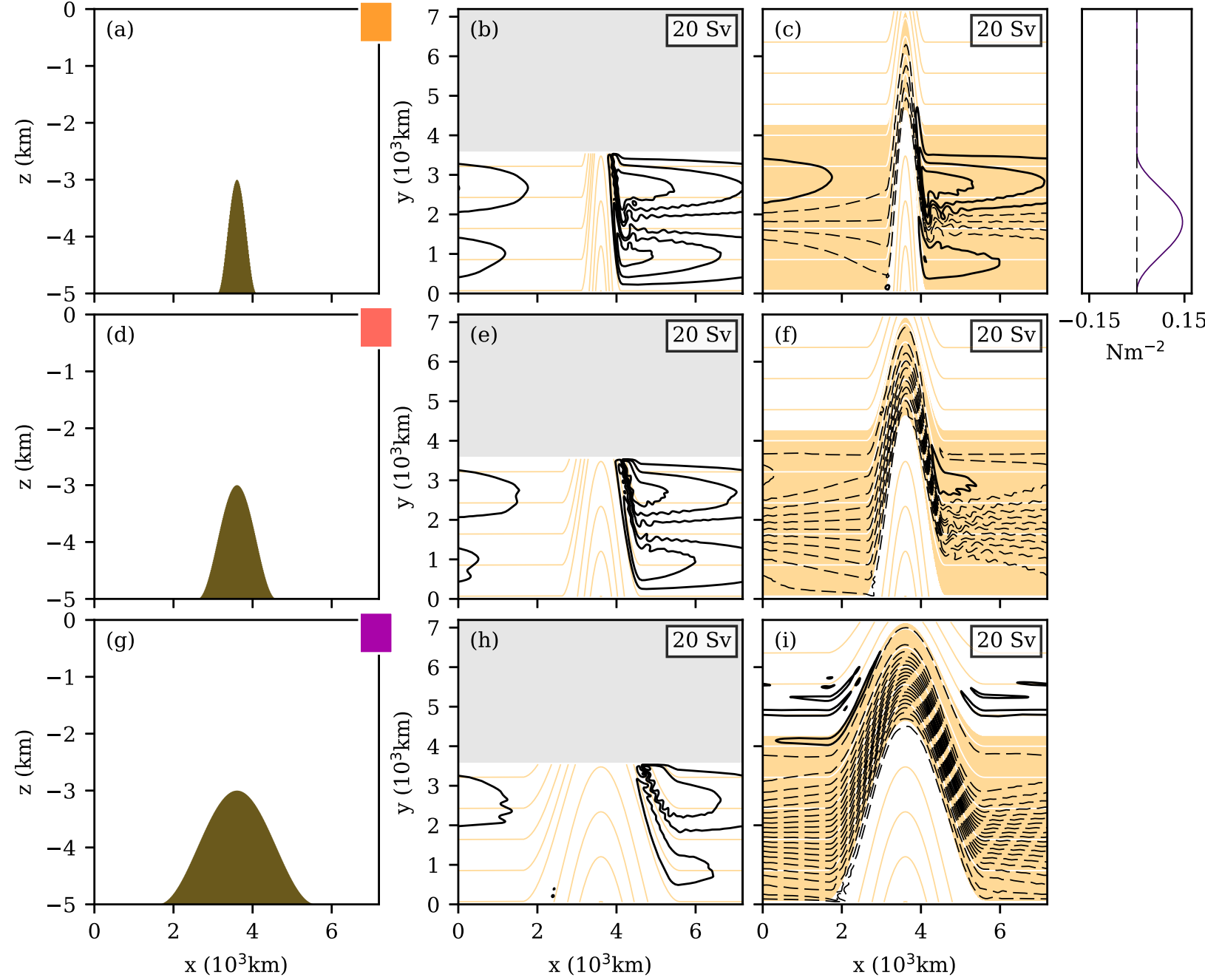

FIG. 7. Plan view of two barotropic channel simulations with variation of the meridional extent. (center) Results for a 2000-m ridge height with 3600-km meridional extent (models 18, 20 and 21, respectively). The gray section signifies land. (right) Results for a 2000-m ridge height with 7200-km meridional extent (models 22, 23 and 24, respectively). The ridge widths are (b),(c) $1000 \mathrm{~km}$, (e),(f) $2000 \mathrm{~km}$, and (h),(i) $4000 \mathrm{~km}$. The plot on the right shows the meridional profile of the zonal wind for each case. The colored boxes in the top-right corners of (a), (d), and (g) are associated with ridge width. For coloring and contouring see Fig. 2. The broken streamlines represent circumpolar flow, and the solid streamlines represent stationary eddies or gyre flow.

creating an experiment whereby topographic gradients are varied without any change in the level of blocked $f / H$. This is achieved through varying ridge width rather than ridge height in a domain with unblocked geostrophic contours.

\section{b. Unblocked geostrophic contours and topography}

The results in Fig. 7 indicate that gyres can form without blocking geostrophic contours. Figure 7b shows the $2000 \mathrm{~m}$ ridge results from Fig. 6. Extending the northern boundary of this case unblocks a large proportion of the geostrophic contours. Previous theory would suggest that a linear free mode would develop and this region of unblocked geostrophic contours would be dominated by circumpolar flow. Contrary to this expectation, sizeable gyres remain. In the extended domain shown by Figs. 7c, 7f, and $7 \mathrm{i}$, increasing ridge width diminishes the gyres. In concurrence with the uniform wind case presented in section 3, the net zonal volume transport in Figs. $7 \mathrm{c}, 7 \mathrm{f}$, and $7 \mathrm{i}$ increases with increased ridge width. The largest ridge width shows very little capability of supporting gyre formation. These results suggest that a submarine ridge can support gyres in regions of unblocked geostrophic contours if the ridge is narrow enough. 


\section{c. The vorticity balance}

The mechanism for the gyres to appear in regions of unblocked geostrophic contours requires a consideration of vorticity. Gyres are absent in results of section 3 due to a lack of curl in the wind forcing, which would induce vorticity. In classical gyre theory, flow is advected across geostrophic contours in the interior of a basin. This interior flow is generated because of a source of vorticity from a curl in the wind stress (Sverdrup 1947). The flow returns meridionally as a narrow western boundary current, closing the circulation (Stommel 1948). This circulation holds in a flat-bottomed, closed basin where geostrophic contours are strictly zonal, intersecting with the western boundary. For the flow to return across geostrophic contours, a sink of vorticity is required. In the earliest example, this vorticity sink is provided by bottom stress curl (Stommel 1948). Later ideas introduce a sloping topography along the western boundary (Holland 1967; Salmon 1992). Over the slope, geostrophic contours are deflected equatorward and flow in the western boundary is quasi meridional as a result (Salmon 1992; Hughes and De Cuevas 2001; Jackson et al. 2006). With the introduction of topography, the point where geostrophic contours intersect a boundary is not necessarily along the western boundary. The influence of topography could result in this position being along a northern or southern boundary and for this reason the position can be labeled the "dynamical west." Although the paths of the geostrophic contours are altered, the vorticity balance is essentially the same. There is an interior source of vorticity from the wind stress which moves the flow across geostrophic contours. Wherever the geostrophic contours intersect with a boundary, flow is advected back to its original value of $f / H$ due to vorticity generated by bottom stress, which is associated with a lateral shear in the flow (Salmon 1992).

Although the gyres in Figs. 7c, 7f, and 7i form in a region of unblocked geostrophic contours, the vorticity balance described above remains. Wind stress curl provides a source of vorticity to flux fluid parcels across geostrophic contours. A sink of vorticity is then required for flow to return across the geostrophic contours and close the circulation. Although classical studies cite bottom stress as the vorticity sink, several terms of the barotropic vorticity Eq. (3) are capable of taking this role. The vorticity sink can arise through the bottom stress curl $\left(-\mathbf{k} \cdot \nabla \times \overline{\boldsymbol{\tau}}_{b}\right)$, eddy inertial term $\left[\rho_{0} \mathbf{k} \cdot \nabla \times\left(\bar{H} \overline{\mathbf{u}^{\prime} \cdot \nabla \mathbf{u}^{\prime}}\right)\right]$, or lateral viscosity $\left(\mu \bar{H} \nabla^{2} \bar{\zeta}\right)($ Jackson et al. 2006). The mean inertial term $[\mathbf{k} \cdot \nabla \times(\bar{H} \overline{\mathbf{u}} \cdot \nabla \overline{\mathbf{u}})]$ can alter the characteristics of a gyre (Fofonoff 1954; Veronis 1966) but cannot provide a net sink of vorticity. Taking an area integral bounded by a closed streamline, $\mathbf{k} \cdot \nabla \times(\bar{H} \overline{\mathbf{u}} \cdot \nabla \overline{\mathbf{u}})$ integrates to zero in a steady ocean (Böning 1986; Jackson et al. 2006).

To investigate the vorticity balance, all terms of the barotropic vorticity equation for the central part of the simulations with unblocked geostrophic contours are shown in Fig. 8. In the absence of topography in a channel, the vorticity input via wind stress curl is locally balanced everywhere by bottom stress curl (not shown). The introduction of topography causes this balance to become nonlocal and much of the wind stress curl is balanced over the topography. The main balance over the topography in Fig. 8 is between the advection of planetary vorticity $\left(-\rho_{0} \bar{H} \beta \bar{v}\right)$ and the bottom pressure torque $\left(\mathbf{k} \cdot \nabla \bar{p}_{b} \times \nabla h\right)$, which is indicative of flow generally following geostrophic contours (Jackson et al. 2006). There is a contribution of vorticity from the mean-inertial term but as discussed above this has no net influence along the entirety of a streamline. The lateral viscosity term is clearly not responsible for the formation of gyres in these experiments as it is negligible in all cases. The vorticity sources from the bottom stress and the eddy inertial terms are nonnegligible for these simulations. Both terms increase as ridge width decreases. The dynamics presented suggest that both eddies and bottom stress could be the balancing terms for the vorticity induced by the wind stress. It will be shown below that the same results hold in the absence of eddies with bottom stress providing a sufficient representation of the dynamics.

\section{d. The details of the bottom stress vorticity sink}

We have shown how the dynamics allow for gyre formation but why gyres form in place of circumpolar flow is yet to be explained. There is an existing suggestion that geostrophic contours are not required to intersect with a boundary for gyres to form in a North Atlantic setting (Salmon 1992). Salmon (1992) outlines that the "frictional" (vorticity) balance within a gyre will be met regardless of the topography. Bottom stress curl is dependent on velocity gradients, which are enhanced where geostrophic contours converge. In a domain where geostrophic contours do not intersect with a boundary, the vorticity balance can be met via the generation of bottom stress curl where gradients in geostrophic contours are largest. As a result, the vorticity sink in the results we present occurs on the ridge slope where geostrophic contours converge and gradients in potential vorticity increase.

A dynamical difference between the North Atlantic and the Southern Ocean exists due to the presence of the ACC. The entire vorticity sink balancing the wind 


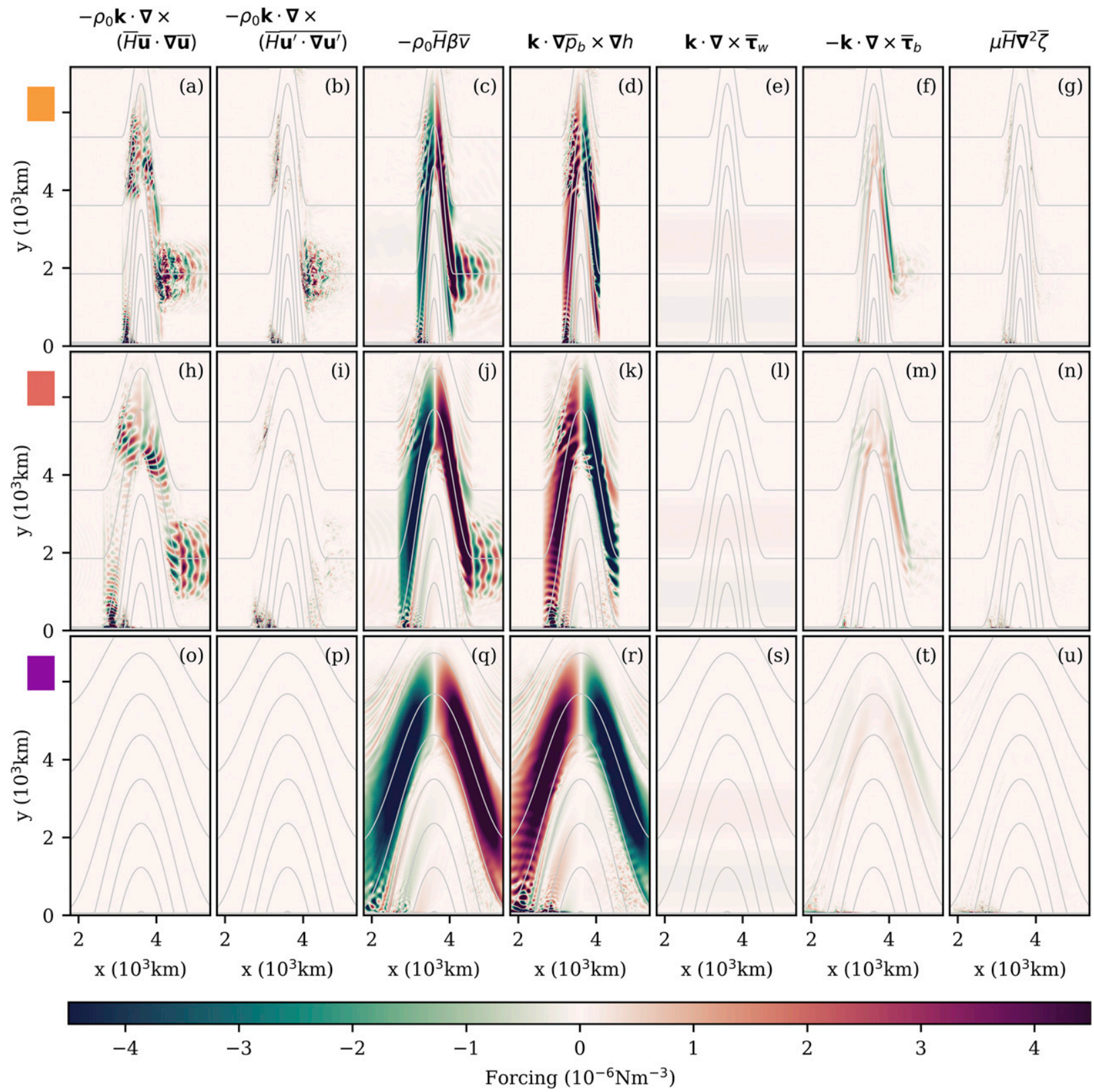

FIG. 8. Terms of the barotropic vorticity equation for the extended simulations shown in Fig. 7 (models 22-24). Simulations have a meridionally aligned ridge of $2000 \mathrm{~m}$ in height and of varying ridge widths. Rows are associated with particular ridge widths (model 22: $1000 \mathrm{~km}$, model 23: $2000 \mathrm{~km}$, and model 24: $4000 \mathrm{~km}$ ). Columns represent differing barotropic vorticity terms: mean inertial term, eddy vorticity flux, advection of planetary vorticity, bottom pressure torque, wind stress curl, bottom stress curl, and viscosity. The colored boxes to the left of (a), (h), and (o) are associated with ridge width.

vorticity source occurs via a western boundary current in the North Atlantic setting, whereas, in the Southern Ocean a proportion of this vorticity sink is accounted for by the ACC. Figure 9 shows the bottom stress curl over the topography for two ridge widths. The dominant dipole that spans the ridge is associated with the convergence of the circumpolar current across the topography (Fig. 9b), an absent characteristic when there is no wind vorticity source (section 3). The bottom stress curl associated with the gyres is more subtle (Figs. 9a,c). Figure 9c shows that the vorticity associated with the upslope gyre flow coincides with streamlines crossing geostrophic contours, directed toward the circumpolar current. The flow joins the circumpolar jet, enhancing the vorticity sink generated in the downslope flow. Unlike classical 


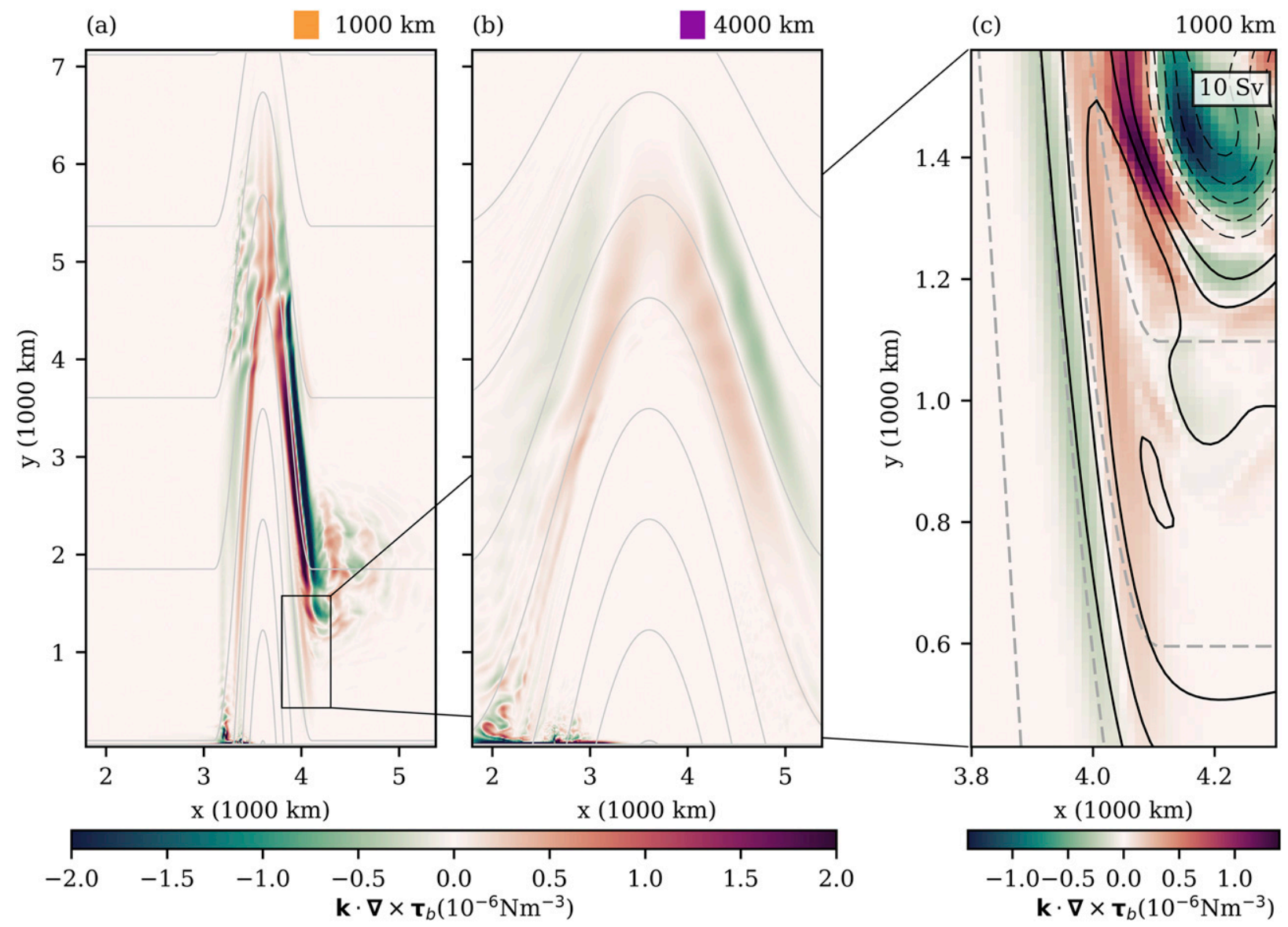

FIG. 9. The $\mathbf{k} \cdot \nabla \times \boldsymbol{\tau}_{b}$ for varying ridge width. Results are for model simulations 22 and 24 shown in Fig. $8 ; \mathbf{k} \cdot \nabla \times \boldsymbol{\tau}_{b}$ is shown for results with ridge width of (a) 1000 and (b) $4000 \mathrm{~km}$. Solid gray lines in (a) and (b) are contours of $f / h$. (c) The $\mathbf{k} \cdot \nabla \times \boldsymbol{\tau}_{b}$ for the $1000-\mathrm{km}$ ridge simulation focused on the eastern flank of the ridge, with streamlines overlaid in black. The dashed black streamlines represent circumpolar flow, and the solid black streamlines represent stationary eddies or gyre flow. Dashed gray lines in (c) are contours of $f / h$. The box in the top-right corner of (c) shows the streamline spacing in Sverdrups. The colored boxes above (a) and (b) are associated with ridge width.

gyre dynamics, both the circumpolar current and the gyres are responsible for balancing the vorticity associated with the wind stress curl.

The results suggest that the gyres become increasingly important in balancing the wind vorticity source as the ridge width and circumpolar transport reduces. The vorticity input via the wind is constant between simulations. A reduction in ridge width induces a vorticity sink associated with the gyres, which indicates a reduction in the vorticity sink associated with the circumpolar current. Thus, gyres form and increase in strength with decreasing ridge width to maintain the vorticity balance when the circumpolar current reduces in strength. Momentum conservation is also important to the gyre results and we will discuss this below.

\section{e. The combined control of vorticity and momentum}

The experiments in this study investigate the dynamics of a nonlinear system. In general, increasing the bottom stress coefficient damps the inertia of a system, reducing the effect of nonlinear terms. Figure 10 shows the response of the gyre and circumpolar transport to an increase in the bottom stress coefficient. As the coefficient is increased, the gyre strength increases for the 1000- and 2000-km-wide simulations and remains unchanged for the 4000-km-wide ridge. The absence of any notable gyre in the $4000-\mathrm{km}$ case shown by Fig. $7 \mathrm{i}$ is the reason for the insensitivity of the gyre transport in this case. Figure 10b shows that, in alignment with results of section 3 , the circumpolar transport decreases for all simulations as the bottom stress coefficient is increased. Figure 11 shows the streamfunction and terms of the barotropic vorticity equation for two 2000-km cases shown in Fig. 10 . With increased bottom drag coefficient, the inertial (nonlinear) terms become negligible, and it is only bottom stress curl that balances the wind stress curl. The fact that the same relationships remain in a more 

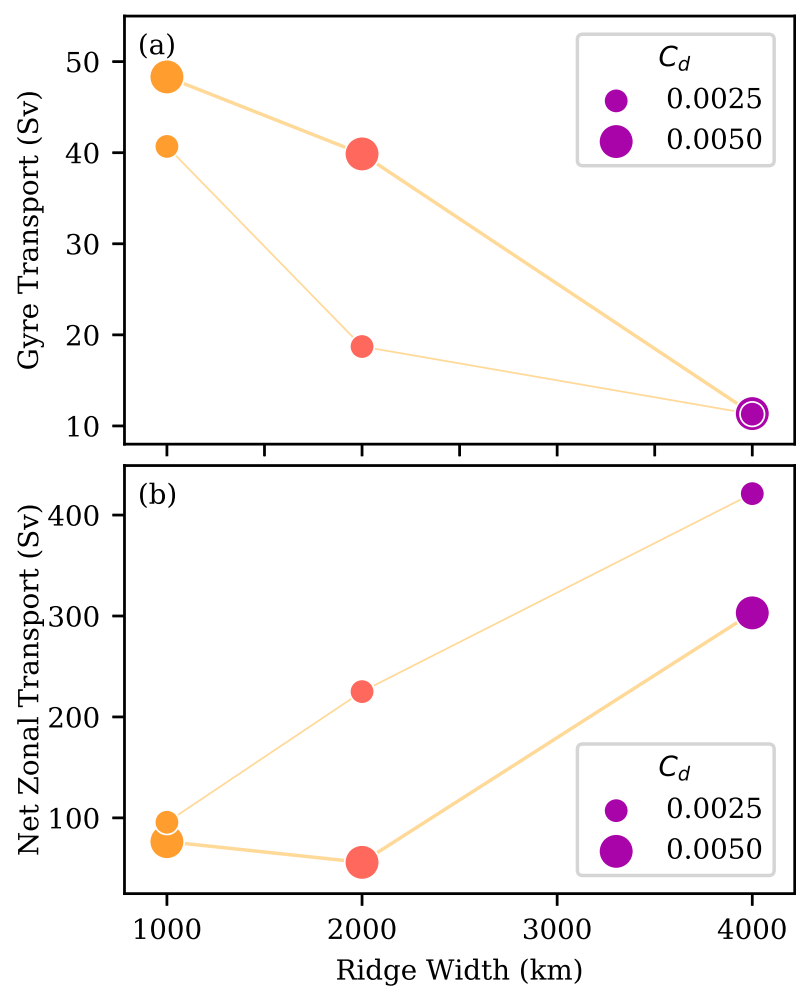

FIG. 10. Volume transport vs ridge width for varying bottom stress coefficient $C_{d}$ of 0.0025 and 0.0050 (models 22-27). The simulations with $C_{d}$ of 0.0025 are the same as presented in Figs. $7 \mathrm{c}, 7 \mathrm{f}, 7 \mathrm{i}$, and 8 . Shown are (a) gyre strength vs ridge width for varying $C_{d}$ and (b) circumpolar transport vs ridge width for varying $C_{d}$.

linear case show that bottom stress alone is capable of generating the required vorticity sink for the formation of gyres in a region of unblocked geostrophic contours.

In the case with a nonzero wind stress curl, the wind stress is a source of both vorticity and momentum and a balance must be achieved for both. In the system presented, bottom stress balances the wind stress in terms of vorticity, which can develop via the circumpolar current and the gyres. In the presence of topography, form stress is the primary balance for the wind stress in terms of momentum. The circumpolar current is not the only route for the generation of form stress across topography. Gyre flow that crosses geostrophic contours along a ridge slope is associated with a pressure difference across the topography (Naveira Garabato et al. 2013). Therefore, gyres are also capable of creating the form stress required to balance the zonal wind stress (Wang and Huang 1995; Naveira Garabato et al. 2013). As gyre transport increases more gyre flow crosses geostrophic contours over the ridge leading to an increase in the form stress associated with the gyres. This indicates that the gyre response not only maintains the balance of vorticity but it also acts to conserve momentum, compensating for changes in form stress associated with the circumpolar current. Figures 10 and 11 highlight that as circumpolar transport reduces, gyres form in order satisfy both vorticity and momentum conservation.

\section{f. The influence of topographic Sverdrup balance}

The focus above has been on the momentum and vorticity sinks that are generated over the topography. The presence of gyres results from the curl in the wind stress. The following results suggest that the change in topography also has an effect on the response of the gyres to the wind forcing.

Gyres are primarily forced by the wind stress curl over the ocean surface. A reduction in the area over which the gyres are forced implies a reduction in gyre strength (Munk 1950). Topographic Sverdrup balance dictates that wind forcing of a gyre is a function of the wind stress curl between bounding geostrophic contours (Holland 1967). In the blocked simulations (Figs. 7b,e,h), the area that the gyres occupy decreases with increased ridge width, indicating that they are being influenced by a changing topographic Sverdrup balance. Figures 12a-f show the wind stress curl over each gyre shown in Figs. 7b, 7e, and 7h. The boundary between the two gyres is the geostrophic contour which lies along the line of zero wind stress curl in the flat bottomed part of the domain. The gyres are also bounded by the domain walls and coincident geostrophic contours in the interior. The gyre bounds are deflected equatorward with the geostrophic contours over the topography. The northern gyre emerges due to positive wind stress curl and the southern gyre is due to negative wind stress curl. In all cases, the area of wind forcing over the gyres reduces as ridge width increases. The southern gyre flows into an area of opposing wind stress curl in the northern part of the domain. As a result, there is an additional sink of vorticity in the northern section of the domain which acts in opposition to the prevailing forcing of this gyre. The area of opposing wind stress curl reduces as ridge width reduces. Figure $12 \mathrm{~g}$ shows the area integral of the wind stress curl over each gyre, normalized against the northern gyre forcing of the narrow ridge simulation. There is a clear reduction in the wind forcing over each gyre as the ridge width is increased. This reduction aligns with the reduction in gyre strength with increased ridge width seen in Fig. 12h. This indicates that there is a sensitivity of gyre strength due to a response of the area of wind forcing to the changes in ridge geometry. 


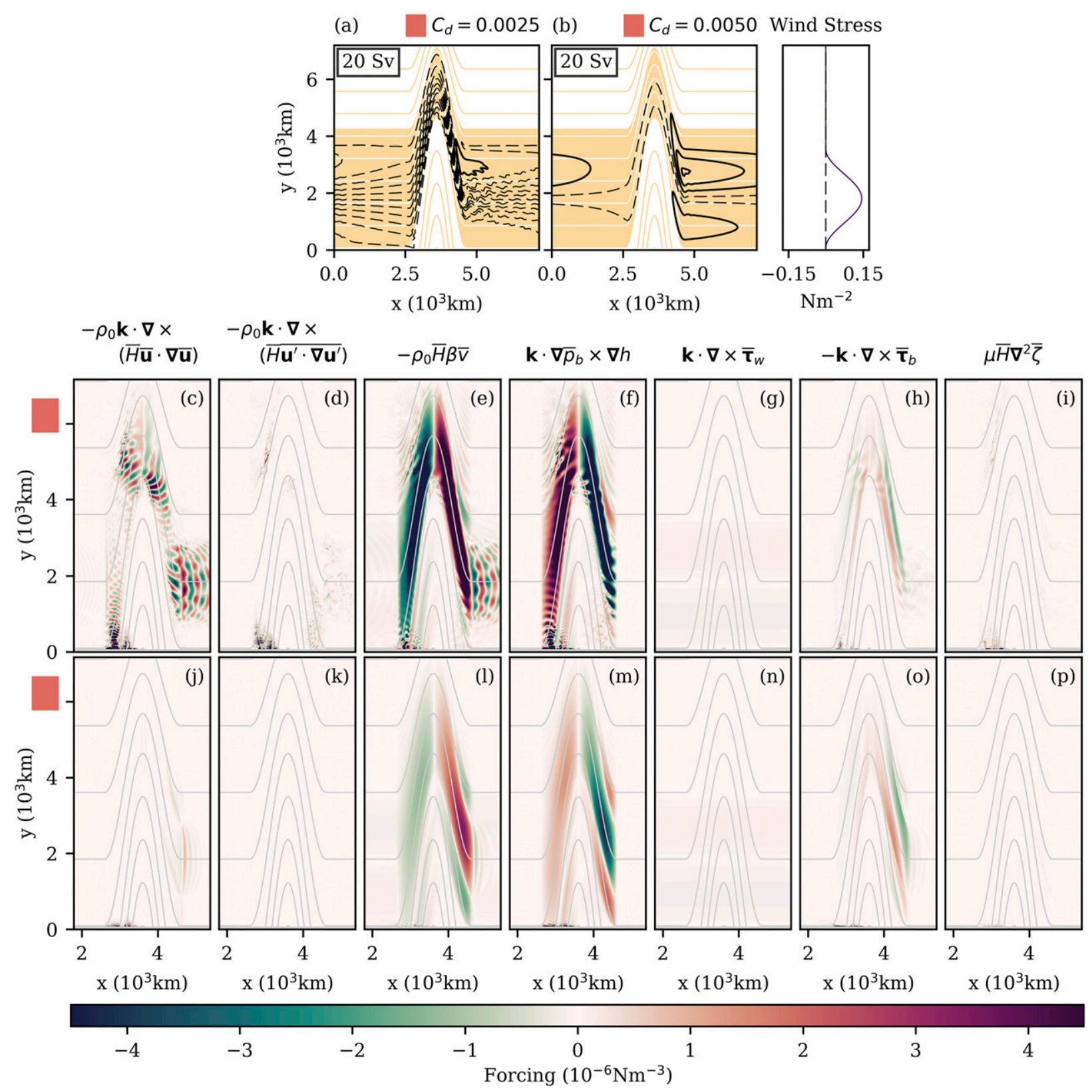

FIG. 11. Barotropic streamfunction and terms of the barotropic vorticity equation for simulations with a meridionally aligned ridge of $2000 \mathrm{~m}$ in height and $2000 \mathrm{~km}$ in width. Results are for model simulations 23 and 26 as shown in Fig. 10 with varying $C_{d}$; shown are the barotropic streamfunction of results with bottom stress coefficient of (a) 0.0025 and (b) 0.0050 . For coloring and contouring see Fig. 2 . The dashed streamlines represent circumpolar flow, and the solid streamlines represent stationary eddies or gyre flow. The remaining plots are associated with a particular bottom stress coefficient: (middle) 0.0025 and (bottom) 0.0050 . Shown are differing barotropic vorticity terms: (c),(j) mean inertial term, (d),(k) eddy vorticity flux, (e),(l) advection of planetary vorticity, (f),(m) bottom pressure torque, (g),(n) wind stress curl, (h),(o) bottom stress curl, and (i),(p) viscosity. The colored boxes above (a) and (b) and to the left of (c) and (j) are associated with ridge width.

The gray lines in Fig. 12h show that the change in gyre strength is much larger in the extended domain in comparison to results in Fig. 12, which has the same wind forcing. Although the topographic Sverdrup balance is shown to have a primary influence on the blocked domain, the difference suggests this is not the case for the extended domain. There is a relationship between ridge width and gyre strength associated with the changes in vorticity and momentum sinks described in the previous sections and changes in 


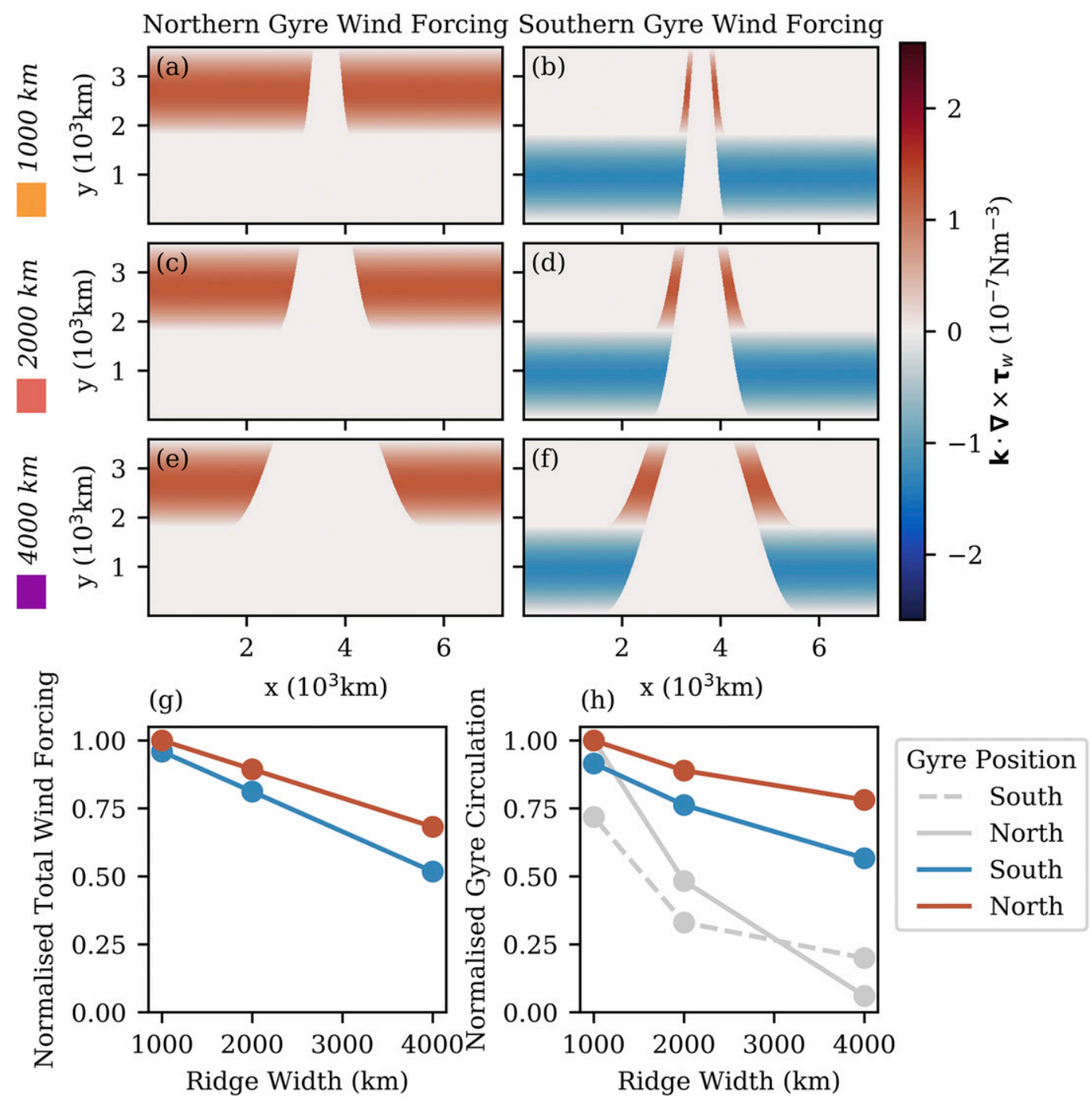

FIG. 12. The wind stress curl over each gyre in Fig. 7b (model 18), Fig. 7e (model 20), and Fig. 7h (model 21). (a)-(f) A plan view of the wind forcing over each gyre. (g) An area integral of the forcing in (a)-(f) normalized to the area integral for (b). (h) The gyre strength vs ridge width. The gray lines in (h) correspond to the normalized gyre strength of models 22-24 where the meridional extent is $7200 \mathrm{~km}$ (See Figs. 7c, 7f, and 7i). The colored boxes to the left of (a), (c), and (e) are associated with ridge width.

topographic Sverdrup balance are indicated to have a second-order effect.

\section{Discussion}

The results presented in this study represent simplified barotropic dynamics. Although the barotropic component of the ACC is not negligible, especially near large topography (Peña-Molino et al. 2014; Donohue et al. 2016), the Southern Ocean is not in the barotropic limit. As a result, some complexities of the Southern Ocean are not represented in our simplified a system. Theories outlined in this paper are intended to enhance our understanding of a more complex, baroclinic eddying system. As such, it is important to discuss how this study might relate to the less idealized setting.

We have investigated the nonlinear system, where topographic form stress is associated with standing meanders in the lee of topography (Johnson 1977; Stevens and Ivchenko 1997). In many parts of the Southern Ocean, baroclinic Rossby waves are suppressed from westward propagation (Hughes et al. 1998) and standing meanders are predominantly associated with Rossby waves of barotropic wavelength (Hughes 2005; Thompson and Naveira Garabato 2014). Despite the fact that barotropic standing meanders are well captured in our results due to nonlinearity, stratification can alter the level of topographic form stress associated with 
particular stationary Rossby waves (Johnson 1977; Stewart and Hogg 2017). With stratification, the balance of momentum remains between wind stress and topographic form stress but the two are not connected directly. The pressure force associated with topographic form stress is thought to communicate through the water column to the bottom topography via interfacial form stress (Johnson and Bryden 1989). The absence of density variations in our barotropic experiments neglects any representation of interfacial form stress and topographic form stress is a function of the sea surface profile alone. The vertical density structure in the ACC can obscure this surface signal reducing the total topographic form stress contribution to the momentum budget (Stewart and Hogg 2017). This reduction in topographic form stress would indicate a larger volume transport than the barotropic counterpart (Stewart and Hogg 2017). As such, stratification could augment results presented in section 3 . However, this effect is sensitive to the particular density structure and it is also possible for this feature of stratification to have the opposite, or even no effect.

Eddying effects are also clearly important to Southern Ocean dynamics (Rintoul 2018) and can be a determining factor of form stress and volume transport. While the simulations presented here show some evidence of eddying motions (for narrow topography in particular), eddies are not a dominant feature. Eddies can influence net zonal volume transport via a processes of "eddy saturation" (Straub 1993), where the ACC is observed to be largely insensitive to changes in the wind forcing because of adjustment in the associated eddy kinetic energy (Meredith and Hogg 2006). This is a process that is observed to occur via both baroclinic and barotropic instabilities (Constantinou and Hogg 2019). Instabilities can feed back on meander dynamics and influence topographic form stress (Naveira Garabato et al. 2014; Youngs et al. 2017). However, stability analysis suggests that the amplitude of the topography we present is too large to exhibit significant barotropic eddy saturation (Hart 1979; Constantinou 2018) and the barotropic instabilities that impact the topographic meander are likely missing. One evident result of the lack in eddy kinetic energy is shown by the contrasting relationship observed in section $3 \mathrm{f}$ to previous studies (Tansley and Marshall 2001; Nadeau and Straub 2012; Nadeau and Ferrari 2015; Marshall et al. 2017). We expect that an increase in eddy kinetic energy in our results would reverse the sensitivity of the ACC's volume transport to changes in the bottom stress coefficient.

Eddying motions also play a role in gyre dynamics. We have confirmed the hypothesis of Nadeau and Ferrari (2015), showing that the required vorticity sink for gyre formation is generated when potential vorticity gradients increase, with no reliance on blocked geostrophic contours. In a barotropic eddy-saturated case, eddies are dominant in diffusing potential vorticity across geostrophic contours (Constantinou 2018). The vorticity sink in our simulations is described in terms of bottom stress but we expect that with a stronger eddying field geostrophic eddies would have a similar effect. An indication of this is given in Fig. 8. The Reynolds stresses are enhanced with reduced topographic width, scaling similarly to bottom stress with changes in ridge topography and diffusing potential vorticity in a similar way. Further evidence points to this relationship remaining comparable in the presence baroclinic instabilities with both barotropic and baroclinic eddies become intensified with increased topographic gradients (Barthel et al. 2017). We speculate that in a highly eddying setting the vorticity dynamics we present will remain, with Reynolds stress superseding the role of bottom stress.

The Southern Ocean is far from the barotropic limit and the theory presented is not directly applicable due to the lack of baroclinicity and strong eddying effects. However, the aim of our investigation is not to give a realistic representation of the Southern Ocean but to derive some simple relationships that can aid our understanding of the complex system that exists. Despite caveats, our results provide insight into the general process by which Southern Ocean gyres form. Further, we have made a contribution in understanding the role of from stress in flow over topography. The dynamics are expected to change with the introduction of stratification and a strong eddy field. We have identified some of the possible consequences of including these effects. Using our study as a basis, it would be useful for future studies to fully investigate how these effects might influence the relationships we present concerning Southern Ocean flow in regions of unblocked geostrophic contours.

\section{Summary}

There are many unexplored aspects of topographically influenced flow. This study has sought to investigate the role of varying geometry on flow in the Southern Ocean. A series of experiments are carried out exploring the response of Southern Ocean gyres and the ACC to variations in the width of a meridionally aligned ridge. Investigation into the direct effects of stratification and eddies has been omitted; it is noted that these are worthy of future investigation. However, our study particularly highlights the dynamical need to balance momentum and vorticity, a general result that would hold regardless or the presence of eddies and baroclinicity. 
Previous work highlights that topography is highly influential on the dynamics of large-scale flow (Munk and Palmén 1951; Hughes and Killworth 1995). It has long been acknowledged that topography has a large influence on the path of the ACC (Gordon et al. 1978; Killworth 1992). Idealized channel simulations have been carried out of flow over topography to gain insight into ACC dynamics. We build on existing studies by investigating nonlinear dynamics in a region of unblocked geostrophic contours. The results show that the volume transport of a barotropic circumpolar current is highly sensitive to ridge width variations. As ridge width is increased there is a large increase in the volume transport. The underlying mechanism for this relationship is shown here to be related to form stress, the primary sink of zonal momentum balancing the zonal wind stress over the Southern Ocean (Munk and Palmén 1951; Wolff et al. 1991; Stevens and Ivchenko 1997; Masich et al. 2015). The steady-state results show that, for different ridge width variations, the form stress remains the primary sink through an adjustment in the SSH profile over the topography. This adjustment is then associated with a change in zonal volume transport with changing ridge width. This indicates that the observed ACC volume transport is determined by the geometry of large-scale topographic features in the Southern Ocean. An additional relationship has also been observed. As ridge width is increased there is a small reduction in the proportion of wind stress which is balanced by form stress in the zonal momentum balance. This reduction is accounted for by an increase in bottom stress. It is shown that this proportional balance is associated with the volume transport and an increased bottom stress coefficient leads to a reduced volume transport as a result.

Established theory bases the formation of Southern Ocean gyres on the intersection of geostrophic contours with a landmass to the dynamical west (LaCasce and Isachsen 2010). It is highlighted here that gyre circulations in the Southern Ocean do not necessarily form in this manner. The Ross Gyre forms in the lee of a topographic feature where all geostrophic contours are unblocked. Established theory would suggest that this space should be occupied by the ACC. This study shows that gyres can occur in the absence of geostrophic contours intersecting with boundaries. In the presence of wind forcing with a nonzero curl, the wind stress must be balanced in terms of both momentum and vorticity. The necessary balances for the wind stress can develop through the dynamics of both gyres and the circumpolar current. Zonal momentum constraints are associated with a reduction in circumpolar volume transport with a reduced ridge width, in turn reducing the bottom stress curl. The bottom stress curl must be maintained to balance the wind stress curl and gyres form in order for this to happen. In addition, there is an auxiliary effect of the ridge width variation on the character of gyres. Because of topographic Sverdrup balance, the change in topography alters the geostrophic contours, which is associated with a reduction in the wind forcing of the gyres. The curtailed wind forcing is linked to a reduction in the gyre strength and a proportion of the relationship between ridge width and gyre strength is linked to topographic Sverdrup balance.

Acknowledgments. We acknowledge insightful comments from Chris Hughes and George Nurser. This work was supported by the Natural Environmental Research Council (Grant NE/L002531/1).

\section{REFERENCES}

Armitage, T. W. K., R. Kwok, A. F. Thompson, and G. Cunningham, 2018: Dynamic topography and sea level anomalies of the Southern Ocean: Variability and teleconnections. J. Geophys. Res. Oceans, 123, 613-630, https://doi.org/10.1002/2017JC013534.

Barthel, A., A. McC. Hogg, S. Waterman, and S. Keating, 2017: Jet-topography interactions affect energy pathways to the deep Southern Ocean. J. Phys. Oceanogr., 47, 1799-1816, https://doi.org/10.1175/JPO-D-16-0220.1.

Böning, C. W., 1986: On the influence of frictional parameterization in wind-driven ocean circulation models. Dyn. Atmos. Oceans, 10, 63-92, https://doi.org/10.1016/0377-0265(86)90010-2.

Constantinou, N. C., 2018: A barotropic model of eddy saturation. J. Phys. Oceanogr., 48, 397-411, https://doi.org/10.1175/ JPO-D-17-0182.1.

— , and A. M. Hogg, 2019: Eddy saturation of the southern ocean: a baroclinic versus barotropic perspective. Geophys. Res. Lett., 46, https://doi.org/10.1029/2019GL084117, in press.

Donohue, K. A., K. L. Tracey, D. R. Watts, M. P. Chidichimo, and T. K. Chereskin, 2016: Mean Antarctic Circumpolar Current transport measured in Drake Passage. Geophys. Res. Lett., $\mathbf{4 3}$ 11760-11 767, https://doi.org/10.1002/2016GL070319.

Fofonoff, N. P., 1954: Steady flow in a frictionless homogeneous ocean. J. Mar. Res., 13, 254-262.

Gordon, A. L., E. Molinelli, and T. Baker, 1978: Large-scale relative dynamic topography of the Southern Ocean. J. Geophys. Res., 83, 3023-3032, https://doi.org/10.1029/JC083iC06p03023.

Hart, J. E., 1979: Barotropic quasi-geostrophic flow over anisotropic mountains. J. Atmos. Sci., 36, 1736-1746, https://doi.org/ 10.1175/1520-0469(1979)036<1736:BQGFOA >2.0.CO;2.

Holland, W. R., 1967: On the wind-driven circulation in an ocean with bottom topography. Tellus, 19, 582-600, https://doi.org/ 10.3402/tellusa.v19i4.9825.

- 1972: Baroclinic and topographic influences on the transport in western boundary currents. Geophys. Astrophys. Fluid Dyn., 4, 187-210, https://doi.org/10.1080/03091927208236095.

Hughes, C. W., 2005: Nonlinear vorticity balance of the Antarctic Circumpolar Current. J. Geophys. Res., 110, C11008, https:// doi.org/10.1029/2004JC002753.

, and P. D. Killworth, 1995: Effects of bottom topography in the large-scale circulation of the Southern Ocean. J. Phys. 
Oceanogr., 25, 2485-2497, https://doi.org/10.1175/1520-0485(1995) 025<2485:EOBTIT $>2.0$. CO; 2 .

—, and E. R. Ash, 2001: Eddy forcing of the mean flow in the Southern Ocean. J. Geophys. Res., 106, 2713-2722, https:// doi.org/10.1029/2000JC900332.

_ , and B. A. De Cuevas, 2001: Why western boundary currents in realistic oceans are inviscid: A link between form stress and bottom pressure torques. J. Phys. Oceanogr., 31, 2871-2885, https://doi.org/10.1175/1520-0485(2001)031<2871:WWBCIR> 2.0.CO;2.

_- M. S. Jones, and S. Carnochan, 1998: Use of transient features to identify eastward currents in the Southern Ocean. J. Geophys. Res., 103, 2929-2943, https://doi.org/ 10.1029/97JC02442.

—, M. P. Meredith, and K. J. Heywood, 1999: Wind-driven transport fluctuations through drake passage: A southern mode. J. Phys. Oceanogr., 29, 1971-1992, https://doi.org/ 10.1175/1520-0485(1999)029<1971:WDTFTD > 2.0.CO;2.

Jackson, L., C. W. Hughes, and R. G. Williams, 2006: Topographic control of basin and channel flows: The role of bottom pressure torques and friction. J. Phys. Oceanogr., 36, 1786-1805, https://doi.org/10.1175/JPO2936.1.

Johnson, E. R., 1977: Stratified Taylor columns on a beta-plane Geophys. Astrophys. Fluid Dyn., 9, 159-177, https://doi.org/ 10.1080/03091927708242323.

Johnson, G. C., and H. L. Bryden, 1989: On the size of the Antarctic Circumpolar Current. Deep-Sea Res., 36A, 39-53, https:// doi.org/10.1016/0198-0149(89)90017-4.

Johnson, J., and R. Hill, 1975: A three-dimensional model of the Southern Ocean with bottom topography. Deep-Sea Res. Oceanogr. Abstr., 22, 745-751, https://doi.org/10.1016/ 0011-7471(75)90079-0.

Killworth, P. D., 1992: An equivalent-barotropic mode in the Fine Resolution Antarctic Model. J. Phys. Oceanogr., 22, 1379-1387, https://doi.org/10.1175/1520-0485(1992) 022<1379:AEBMIT>2.0.CO;2.

Krupitsky, A., and M. A. Cane, 1994: On topographic pressure drag in a zonal channel. J. Mar. Res., 52, 1-23, https://doi.org/ 10.1357/0022240943076740.

_- V. M. Kamenkovich, N. Naik, and M. A. Cane, 1996: A linear equivalent barotropic model of the Antarctic Circumpolar Current with realistic coastlines and bottom topography. J. Phys. Oceanogr., 26, 1803-1824, https://doi.org/10.1175/ 1520-0485(1996)026<1803:ALEBMO>2.0.CO;2.

LaCasce, J., and P. Isachsen, 2010: The linear models of the ACC. Prog. Oceanogr., 84, 139-157, https://doi.org/10.1016/ j.pocean.2009.11.002.

Marshall, D., 1995: Topographic steering of the Antarctic Circumpolar Current. J. Phys. Oceanogr., 25, 1636-1650, https://doi.org/ 10.1175/1520-0485(1995)025<1636:TSOTAC >2.0.CO;2.

- M. H. P. Ambaum, J. R. Maddison, D. R. Munday, and L. Novak, 2017: Eddy saturation and frictional control of the Antarctic Circumpolar Current. Geophys. Res. Lett., 44, 286292, https://doi.org/10.1002/2016GL071702.

Marshall, J., A. Adcroft, C. Hill, L. Perelman, and C. Heisey, 1997a: A finite-volume, incompressible Navier Stokes model for studies of the ocean on parallel computers. J. Geophys Res., 102, 5753-5766, https://doi.org/10.1029/96JC02775.

_ C. Hill, L. Perelman, and A. Adcroft, 1997b: Hydrostatic, quasihydrostatic, and nonhydrostatic ocean modeling. J. Geophys. Res., 102, 5733-5752, https://doi.org/10.1029/96JC02776.

Masich, J., T. K. Chereskin, and M. R. Mazloff, 2015: Topographic form stress in the Southern Ocean State Estimate.
J. Geophys. Res. Oceans, 120, 7919-7933, https://doi.org/ 10.1002/2015JC011143.

McCartney, M., 1976: The interaction of zonal currents with topography with applications to the Southern Ocean. Deep-Sea Res. Oceanogr. Abstr., 23, 413-427, https://doi.org/10.1016/ 0011-7471(76)90838-X.

Meredith, M. P., and A. M. Hogg, 2006: Circumpolar response of Southern Ocean eddy activity to a change in the Southern Annular Mode. Geophys. Res. Lett., 33, L16608, https:// doi.org/10.1029/2006GL026499.

Munday, D. R., H. L. Johnson, and D. P. Marshall, 2015: The role of ocean gateways in the dynamics and sensitivity to wind stress of the early Antarctic Circumpolar Current. Paleoceanography, 30, 284-302, https://doi.org/10.1002/2014PA002675.

Munk, W. H., 1950: On the wind-driven ocean circulation. J. Meteor., 7, 80-93, https://doi.org/10.1175/1520-0469(1950) $007<0080$ :OTWDOC $>2.0$.CO;2.

—_ and E. Palmén, 1951: Note on the dynamics of the Antarctic Circumpolar Current. Tellus, 3, 53-55, https://doi.org/10.3402/ tellusa.v3i1.8609.

Nadeau, L.-P., and R. Ferrari, 2015: The role of closed gyres in setting the zonal transport of the Antarctic Circumpolar Current. J. Phys. Oceanogr., 45, 1491-1509, https://doi.org/10.1175/JPO-D-14-0173.1.

— , and D. N. Straub, 2012: Influence of wind stress, wind stress curl, and bottom friction on the transport of a model Antarctic Circumpolar Current. J. Phys. Oceanogr., 42, 207-222, https:// doi.org/10.1175/JPO-D-11-058.1.

Naveira Garabato, A. C., A. G. Nurser, R. B. Scott, and J. A. Goff, 2013: The impact of small-scale topography on the dynamical balance of the ocean. J. Phys. Oceanogr., 43, 647-668, https:// doi.org/10.1175/JPO-D-12-056.1.

_ A. P. Williams, and S. Bacon, 2014: The three-dimensional overturning circulation of the Southern Ocean during the WOCE era. Prog. Oceanogr., 120, 41-78, https://doi.org/ 10.1016/j.pocean.2013.07.018.

Orsi, A. H., T. Whitworth, and W. D. Nowlin, 1995: On the meridional extent and fronts of the Antarctic Circumpolar Current. Deep-Sea Res. I, 42, 641-673, https://doi.org/10.1016/ 0967-0637(95)00021-W.

Patmore, R. D., 2018: Topographic control of Southern Ocean gyres and the Antarctic Circumpolar Current. Ph.D. dissertation, University of Southampton, $176 \mathrm{pp}$.

Peña-Molino, B., S. R. Rintoul, and M. R. Mazloff, 2014: Barotropic and baroclinic contributions to along-stream and across-stream transport in the Antarctic Circumpolar Current. J. Geophys. Res. Oceans, 119, 8011-8028, https://doi.org/ 10.1002/2014JC010020.

Read, P., P. Rhines, and A. White, 1986: Geostrophic scatter diagrams and potential vorticity dynamics. J. Atmos. Sci., 43, 3226-3240, https://doi.org/10.1175/1520-0469(1986) 043<3226:GSDAPV>2.0.CO;2.

Rintoul, S. R., 2018: The global influence of localized dynamics in the southern ocean. Nature, 558, 209, https://doi.org/10.1038/ s41586-018-0182-3.

Rossby, C.-G., 1936: Dynamics of steady ocean currents in the light of experimental fluid mechanics. Massachusetts Institute of Technology and Woods Hole Oceanographic Institution Papers in Physical Oceanography and Meteorology, Vol. 5, No. 1, 43 pp. , and Coauthors, 1940: Planetary flow patterns in the atmosphere. Quart. J. Roy. Meteor. Soc., 66, 68-87.

Salmon, R., 1992: A two-layer Gulf Stream over a continental slope. J. Mar. Res., 50, 341-365, https://doi.org/10.1357/ 002224092784797610. 
Speer, K., S. R. Rintoul, and B. Sloyan, 2000: The diabatic Deacon cell. J. Phys. Oceanogr., 30, 3212-3222, https://doi.org/10.1175/ 1520-0485(2000)030<3212:TDDC >2.0.CO;2.

Stevens, D. P., and V. O. Ivchenko, 1997: The zonal momentum balance in an eddy-resolving general-circulation model of the Southern Ocean. Quart. J. Roy. Meteor. Soc., 123, 929-951, https://doi.org/10.1002/qj.49712354008.

Stewart, A. L., and A. M. Hogg, 2017: Reshaping the Antarctic Circumpolar Current via Antarctic Bottom Water export. J. Phys. Oceanogr., 47, 2577-2601, https://doi.org/10.1175/JPO-D-17-0007.1.

Stommel, H., 1948: The westward intensification of wind-driven ocean currents. Eos, Trans. Amer. Geophys. Union, 29, 202-206, https://doi.org/10.1029/TR029i002p00202.

Straub, D. N., 1993: On the transport and angular momentum balance of channel models of the Antarctic Circumpolar Current. J. Phys. Oceanogr., 23, 776-782, https://doi.org/ 10.1175/1520-0485(1993)023<0776:OTTAAM > 2.0.CO;2.

Sverdrup, H. U., 1947: Wind-driven currents in a baroclinic ocean; With application to the equatorial currents of the eastern Pacific. Proc. Natl. Acad. Sci. USA, 33, 318-326, https:// doi.org/10.1073/pnas.33.11.318.

Talley, L. D., 2013: Closure of the global overturning circulation through the Indian, Pacific, and Southern oceans: Schematics and transports. Oceanography, 26 (1), 80-97, https://doi.org/ 10.5670/OCEANOG.2013.07.

Tansley, C. E., and D. P. Marshall, 2001: On the dynamics of wind-driven circumpolar currents. J. Phys. Oceanogr., 31, 3258-3273, https://doi.org/10.1175/1520-0485(2001) $031<3258$ :OTDOWD $>2.0 . \mathrm{CO} ; 2$.
Thompson, A. F., and K. J. Richards, 2011: Low frequency variability of Southern Ocean jets. J. Geophys. Res., 116, C09022, https://doi.org/10.1029/2010JC006749.

—_, and A. C. Naveira Garabato, 2014: Equilibration of the Antarctic Circumpolar Current by standing meanders. J. Phys. Oceanogr., 44, 1811-1828, https://doi.org/10.1175/ JPO-D-13-0163.1.

Treguier, A. M., and J. C. McWilliams, 1990: Topographic influences on wind-driven, stratified flow in a $\beta$-plane channel: An idealized model for the Antarctic Circumpolar Current. J. Phys. Oceanogr., 20, 321-343, https://doi.org/10.1175/ 1520-0485(1990)020<0321:TIOWDS >2.0.CO;2.

Veronis, G., 1966: Wind-driven ocean circulation-Part 2. Numerical solutions of the non-linear problem. Deep-Sea Res. Oceanogr. Abstr., 13, 31-55, https://doi.org/10.1016/00117471(66)90004-0.

Wang, L., and R. X. Huang, 1995: A linear homogeneous model of wind-driven circulation in a $\beta$-plane channel. J. Phys. Oceanogr., 25, 587-603, https://doi.org/10.1175/1520-0485(1995) $025<0587$ :ALHMOW $>2.0$.CO;2.

Wolff, J.-O., E. Maier-Reimer, and D. J. Olbers, 1991: Wind-driven flow over topography in a zonal $\beta$-plane channel: A quasigeostrophic model of the Antarctic Circumpolar Current. J. Phys. Oceanogr., 21, 236-264, https://doi.org/10.1175/ 1520-0485(1991)021<0236:WDFOTI >2.0.CO;2.

Youngs, M. K., A. F. Thompson, A. Lazar, and K. J. Richards, 2017: ACC meanders, energy transfer, and mixed barotropicbaroclinic instability. J. Phys. Oceanogr., 47, 1291-1305, https://doi.org/10.1175/JPO-D-16-0160.1. 